\author{
Accelerator Division \\ Alternating Gradient Synchrotron Department \\ BROOKHAVEN NATIONAI LABORATORY \\ Upton, New York 11973 \\ Accelerator Division \\ Technical Note \\ AGS/AD/Tech. Note No. 344
}

\title{
Beam Ellipse Transport in the HITL
}

\author{
C. J. Gardner
}

October 12, 1990

Following is a review of beam ellipse transport in the Heavy Ion Transfer Line (HITL) which currently links the Tandem van de Graaff and the AGS. Sections 1-3 review some basic optics principles. Section 4 is an overview of the line. Sections 5-8 discuss beam ellipse transport in various sections of the line.

\section{Beam Ellipse Optics}

\subsection{The Beam Ellipse}

Let $x_{0}$ and $x_{0}^{\prime}$ be the position and angle of a beam particle with respect to the reference trajectory at a point $s_{0}$ along a beam line. Then the position, $x$, and angle, $x^{\prime}$, of the particle (with respect to the reference trajectory) at the point $s$ along the beam line are given by

$$
\mathbf{Z}=\mathbf{M} \mathbf{Z}_{0}
$$

where

$$
\mathbf{Z}=\left(\begin{array}{l}
\boldsymbol{x} \\
\boldsymbol{x}^{\prime}
\end{array}\right), \quad \mathbf{Z}_{0}=\left(\begin{array}{l}
x_{0} \\
x_{0}^{\prime}
\end{array}\right)
$$

and

$$
\mathbf{M}=\left(\begin{array}{ll}
M_{11} & M_{12} \\
M_{21} & M_{22}
\end{array}\right), \quad|\mathbf{M}|=\mathbf{M}_{11} \mathbf{M}_{22}-\mathbf{M}_{12} \mathbf{M}_{21}=1
$$

is the transfer matrix between $s_{0}$ and $s$,

Now suppose that the beam at $s_{0}$ is contained within the ellipse

$$
\gamma_{0} x_{0}^{2}+2 \alpha_{0} x_{0} x_{0}^{\prime}+\beta_{0} x_{0}^{\prime 2}=\epsilon
$$


where

$$
\beta_{0} \gamma_{0}-\alpha_{0}^{2}=1,
$$

and $\pi \epsilon$ is the area of the ellipse. Let us determine how the region contained in this ellipse is transformed in going from $s_{0}$ to $s$. To do so we write equation (4) in matrix form:

$$
\mathbf{Z}_{0}^{\dagger} \mathbf{E}_{0}^{-1} \mathbf{Z}_{0}=\epsilon, \quad \mathbf{E}_{0}=\left(\begin{array}{rr}
\beta_{0} & -\alpha_{0} \\
-\alpha_{0} & \gamma_{0}
\end{array}\right),
$$

where $a \dagger$ denotes the transpose of the vector or matrix. The matrix $\mathbf{E}_{0}$ is symmetric and has unit determinant:

$$
\mathbf{E}_{0}^{\dagger}=\mathbb{E}_{0}, \quad\left|\mathbf{E}_{0}\right|=\beta_{0} \gamma_{0}-\alpha_{0}^{2}=1
$$

Now using (1) in (6) we find

$$
\mathbf{Z}^{\dagger} \mathbf{E}^{-1} \mathbf{Z}=\epsilon, \quad \mathbf{E}=\mathbf{M} \mathbf{E}_{0} \mathbf{M}^{\dagger}
$$

and it follows from (7) and (3) that

$$
\mathbf{E}^{\dagger}=\mathbf{M} \mathbf{E}_{0}^{\dagger} \mathbf{M}^{\dagger}=\mathbf{M E}_{0} \mathbf{M}^{\dagger}=\mathbf{E}, \quad|\mathbf{E}|=|\mathbf{M}|\left|\mathbf{E}_{0} \| \mathbf{M}^{\dagger}\right|=1
$$

Thus, $\mathbf{E}$ is symmetric and has unit determinant, and we can write

$$
\mathbf{E}=\left(\begin{array}{rr}
\beta & -\alpha \\
-\alpha & \gamma
\end{array}\right), \quad \beta \gamma-\alpha^{2}=1
$$

It follows that the ellipse defined by $\mathbf{E}_{0}$ is transformed into another ellipse defined by $\mathbf{E}=\mathbf{M E}_{0} \mathbf{M}^{\dagger}$ in going from $s_{0}$ to $s$. Now the area, $\pi \epsilon$, enclosed by the ellipses is conserved, and any particle inside (outside) the ellipse at $s_{0}$ will also be inside (outside) the ellipse at $s$. The beam ellipse-i.e. the (smallest) ellipse which contains the particles in the beam-therefore provides a convenient way of keeping track of the particles as they travel along a beam line. Thus, instead of using equation (1) to track each particle as it moves along the beam line, we use the second of equations (8) to determine the evolution of the beam ellipse.

A beam ellipse defined by

$$
\gamma x^{2}+2 \alpha x x^{\prime}+\beta x^{\prime 2}=\epsilon
$$


is shown in Figure 1. Expressing (11) as

$$
\epsilon \beta=x^{2}+\left(\alpha x+\beta x^{\prime}\right)^{2},
$$

and as

$$
\epsilon \gamma=x^{2}+\left(\gamma x+\alpha x^{\prime}\right)^{2}
$$

we see that the maximum $x$ and $x^{\prime}$ of the ellipse are given by $\sqrt{\beta \epsilon}$ and $\sqrt{\gamma \epsilon}$ respectively. Thus $\sqrt{\beta \epsilon}$ and $\sqrt{\gamma \epsilon}$ define the width and the divergence of the beam. The evolution of $\beta$ and the other ellipse parameters along the beam line can be obtained by multiplying the matricies in equation (8):

$$
\begin{gathered}
\beta=M_{11}^{2} \beta_{0}-2 M_{11} M_{12} \alpha_{0}+M_{12}^{2} \gamma_{0}, \\
\alpha=-M_{11} M_{21} \beta_{0}+\left(1+2 M_{12} M_{21}\right) \alpha_{0}-M_{12} M_{22} \gamma_{0} \\
\gamma=M_{21}^{2} \beta_{0}-2 M_{21} M_{22} \alpha_{0}+M_{22}^{2} \gamma_{0} .
\end{gathered}
$$

We note that here we have considered the evolution of the beam ellipse in one plane only. As discussed in reference (2) the beam particles are in fact contained within a six-dimensional ellipsoid characterized by a real, symmetric, positive definite six-by-six matrix $\mathbf{E}$. The evolution of this ellipsoid is given by the second of equations (8) with the two-by-two matrix, $M$, replaced by the six-by-six transfer matrix. The extent of the beam in each dimension, $\mathrm{i}$, is given by $\sqrt{\epsilon E_{i i}}$, where $E_{i i}$ are the diagonal elements of $\mathbf{E}$.

\subsection{Ellipse Evolution in a Drift}

Here we consider the evolution of the beam ellipse in a drift from $s_{0}$ to $s$. The transfer matrix is given by

$$
\mathbf{M}=\left(\begin{array}{cc}
1 & s-s_{0} \\
0 & 1
\end{array}\right),
$$

which when inserted into equations (14-16) yields

$$
\begin{gathered}
\beta(s)=\beta_{0}-2\left(s-s_{0}\right) \alpha_{0}+\left(s-s_{0}\right)^{2} \gamma_{0} \\
\alpha(s)=\alpha_{0}-\left(s-s_{0}\right) \gamma_{0} \\
\gamma(s)=\gamma_{0} .
\end{gathered}
$$


Thus the width of the beam varies in a drift, but the divergence remains constant.

Differentiating (18) and (19) with respect to $s$ we find

$$
\begin{gathered}
\beta^{\prime}(s)=-2 \alpha_{0}+2\left(s-s_{0}\right) \gamma_{0}=-2 \alpha(s), \\
\beta^{\prime \prime}(s)=2 \gamma_{0} .
\end{gathered}
$$

Since $\gamma$ is always positive, it follows that in a drift $\beta(s)$ reaches a minimum value-i.e. a waist-where $\alpha(s)=0$.

\subsection{Ellipse Evolution in a Thin Lens}

When the transfer matrix

$$
\mathbf{M}=\left(\begin{array}{cc}
1 & 0 \\
-1 / F & 1
\end{array}\right)
$$

for a thin lens with focal length, $F$, is inserted into equations (14-16) one finds

$$
\begin{gathered}
\beta=\beta_{0} \\
\alpha=\alpha_{0}+\beta_{0} / F \\
\gamma=\beta_{0} / F^{2}+2 \alpha_{0} / F+\gamma_{0} .
\end{gathered}
$$

Here we see that $\beta$, and hence the width of the beam, are not changed by the thin lens. However $\alpha$ and $\gamma$, and hence the divergence, do change. Equation (25) shows that the change in $\alpha$ is proportional to $\beta_{0}$ and inversely proportional to the focal length. This is analogus to the formula

$$
x^{\prime}=x_{0}^{\prime}-x_{0} / \dot{F}^{\prime}
$$

for the change in $x^{\prime}$ in a thin lens.

\subsection{Point-to-Point Imaging}

Now suppose we have a transfer matrix, $M$, with element $M_{12}=0$. This matrix is of the form

$$
\mathbf{M}=\left(\begin{array}{cc}
m & 0 \\
a & 1 / m,
\end{array}\right)
$$


where $m$ is called the magnification. Inserting this matrix into equation (1) we find

$$
x=m x_{0}, \quad x^{\prime}=a x_{0}+x_{0}^{\prime} / m .
$$

Here we see that the image, $x$, of $x_{0}$ is independent of $x_{0}^{\prime}$ and hence the matrix is said to image a point to a point. The first of equations (29) shows that the image is magnified by the factor $m$.

Inserting the matrix, $M$, into equations (14-16) we find

$$
\begin{gathered}
\beta=m^{2} \beta_{0} \\
\alpha=\alpha_{0}-a m \beta_{0} \\
\gamma=\gamma_{0} / m^{2}-2 a \alpha_{0} / m+a^{2} \beta_{0} .
\end{gathered}
$$

Thus the beam width is magnified by the factor $m$. If $m=1$ then $\beta=\beta_{0}$ and the width of the beam remains unchanged. However the divergence of the beam does change if $a$ is not zero.

\subsection{Waist-to-Waist Imaging}

If the transfer matrix, $M$, is such that a beam ellipse with a waist at $s_{0}$ evolves into a beam ellipse with a waist at $s$, then the matrix is said to image a waist to a waist. The conditions for which this occurs-i.e. the conditions for waist-to-waist imaging-are obtained by setting $\alpha=\alpha_{0}=0$ in equations (14-16). Doing this we find

$$
\beta_{0}^{2}=-\frac{M_{12} M_{22}}{M_{11} M_{21}}, \quad \beta^{2}=-\frac{M_{12} M_{11}}{M_{22} M_{21}} .
$$

Here we see that unlike the condition, $M_{12}=0$, for point-to-point imaging, which is independent of the ellipse parameters, the condition for waist-to-waist imaging depends on the ellipse parameter $\beta_{0}$. If we impose the additional condition, $M_{11}^{2}=M_{22}^{2}$, then it follows from (33) that $\beta=\beta_{0}$. In this case we also have $\gamma=\gamma_{0}$ and hence both the width and divergence of the beam are unchanged. This is an advantage over the point-to-point case in which the divergence does, in general, change even when $\beta=\beta_{0}$. It should also be noted that waist-to-waist imaging is not possible when the condition for point-to-point imaging is satisfied (and $M_{21} \neq 0$ ). In this case equation (33) implies that $\beta_{0}=0$ and the divergence of the beam is infinite. 

the pipe. To determine the acceptance, we need to find ellipse parameters at the pipe's entrance such that $\beta_{\max }$ is as small as possible. This requires that $\beta(s)$ reach a waist half way through the pipe-i.e. at $s=L / 2$-in which case we have $\beta(0)=\beta(L)=\beta_{\max }$. Let us determine the value of $W=\beta(L / 2)$ for which $\beta_{\max }$ is smallest. Using $\alpha(L / 2)=0$ and $\gamma(L / 2)=1 / W$ in $(18)$ and $(19)$ we have

$$
\beta(s)=W+\frac{(s-L / 2)^{2}}{W}, \quad \beta_{\max }=\beta(0)=\beta(L)=W+\frac{L^{2}}{4 W},
$$

and

$$
\alpha(s)=-(s-L / 2) / W, \quad \alpha(0)=\frac{L}{2 W}, \quad \alpha(L)=-\frac{L}{2 W} .
$$

Then differentiating $\beta_{\max }$ with respect to $W$ we find

$$
\frac{d \beta_{\max }}{d W}=1-\frac{L^{2}}{4 W^{2}}, \quad \frac{d^{2} \beta_{\max }}{d W^{2}}=\frac{L^{2}}{2 W^{3}}>0 .
$$

Thus $\beta_{\max }$ is smallest when $W=L / 2$, in which case we have

$$
\beta(0)=\beta(L)=\beta_{\max }=L, \quad \alpha(0)=1, \quad \alpha(L)=-1 .
$$

The acceptance of the pipe is then

$$
\pi \epsilon=\pi a^{2} / L
$$

with $\alpha(0)=1$ and $\beta(0)=L$ at the pipe's entrance.

Now suppose we place a thin focusing lens with focal length

$$
F=\beta(L) / 2=L / 2
$$

at the end of the beam pipe. It follows from (25) that the lens will change $\alpha$ from -1 to 1 and we can therefore transport the beam through another section of pipe with half-aperture $a$ and length $L$. Continuing in this way we can build a beam line and transport the beam as far as we like. If we want to increase the acceptance of the beam line we must decrease the length, $L$, of the sections of pipe and decrease the focal lengths of the lenses accordingly. This, of course, only works in one plane. In the other plane the lenses are defocusing and the beam eventually becomes too large to be contained in the beam pipe. In order to transport the beam an arbitrary distance in both planes one must use both focusing and defocusing lenses. 


\subsection{Beam Ellipse Transport with a Symmetric Doublet Cell}

One scheme for transporting the beam ellipse makes use of quadrupole doublets. A doublet consists of a focusing and a defocusing quadrupole separated by a distance, $d$, which is usually small compared to the focal lengths of the lenses. We call the arrangement of two doublets shown in Figure 2 a symmetric doublet cell. The cell runs from $s=0$ to $s=2(2 l+d)$ and the labels QF and QD denote horizontal focusing and defocusing quadrupoles. The quadrupoles are all excited with the same current and the cell is symmetric about its center, $s=2 l+d$.

Let

$$
\mathrm{M}=\left(\begin{array}{ll}
M_{11} & M_{12} \\
M_{21} & M_{22}
\end{array}\right)
$$

be the transfer matrix from $s=0$ to $s=2 l+d$. Since the cell is symmetric about its center, the transfer matrix from $s=2 l+d$ to $s=2(2 l+d)$ is then

$$
\mathrm{N}=\sigma \mathbf{M}^{\dagger} \sigma=\left(\begin{array}{ll}
M_{22} & M_{12} \\
M_{21} & M_{11}
\end{array}\right)
$$

and the total transfer matrix for the cell is

$$
\mathbf{T}=\mathrm{NM}=\left(\begin{array}{cc}
M_{11} M_{22}+M_{12} M_{21} & 2 M_{12} M_{22} \\
2 M_{11} M_{21} & M_{11} M_{22}+M_{12} M_{21}
\end{array}\right) .
$$

The phase advance, $\psi$, of the cell is given by

$$
\cos \psi=\left(T_{11}+T_{22}\right) / 2=M_{11} M_{22}+M_{12} M_{21}
$$

with the requirement that $\sin \psi$ have the same sign as $T_{12}$.

Now let $\left(\alpha_{0}, \beta_{0}\right)$ and $(\alpha, \beta)$ be the beam ellipse parameters at the beginning and end of the cell. In order to maximize the beam emittance that can pass through the cell, we require that the beam ellipse have a waist half way between the two doublets. If we also require that the beam ellipse have the same parameters at the beginning and end of the cell, then it follows from the symmetry theorem of section 1.6 that $\alpha_{0}=\alpha=0$. Thus $\beta_{0}$ and the transfer matrix $M$ must satisfy the condition for waist-to-waist imaging:

$$
\beta_{0}=\left(-\frac{M_{12} M_{22}}{M_{11} M_{21}}\right)^{1 / 2}
$$


Since an ellipse which enters the cell with parameters $\alpha_{0}=0$ and $\beta_{0}$ given by (49) leaves the cell with the same parameters, one can transport the ellipse through an arbitrary number of such cells. In the horizontal plane the $\beta$ parameter of this ellipse reaches a maximum value, $\beta_{\max }$, in the QF quadrupoles. In the vertical plane the same maximum is reached in the QD quadrupoles. The acceptance of the cell will be largest when $\beta_{\max }$ (which depends on $d, l$, and the strength of the quadrupoles) is smallest.

\subsection{Beam Ellipse Transport with a FODO Cell}

Another scheme for transporting the beam ellipse is the FODO arrangement shown in. Figure 3. The cell runs from $s=0$, which is the center of the first quadrupole, to $s=2 L$, which is the center of the last quadrupole. The quadrupoles are spaced a distance $L$ apart (from center to center), and the labels QF and QD denote horizontal focusing and defocusing quadrupoles. The quadrupoles are all excited with the same current and the cell is symmetric about its center, $s=L$.

If we require that the beam ellipse have the same parameters at the beginning and end of the cell, then it follows from the symmetry of the cell that $\alpha$ must be zero at the center of each quadrupole. At the begining of the cell $\beta$ is given by equation (49) where $M_{i j}$ are the elements of the transfer matrix from the beginning to the center of the cell.

In the horizontal plane $\beta$ reaches a maximum value,

$$
\beta_{\max }=\left(-\frac{M_{12} M_{11}}{M_{22} M_{21}}\right)^{1 / 2}
$$

in the QF quadrupoles. In the vertical plane the same maximum is reached in the QD quadrupoles. The acceptance of the cell will be largest when $\beta_{\max }$ (which depends on $L$ and the strength of the quadrupoles) is smallest.

\section{Bending Magnets with Pole Face Rotation}

In reference (1) it is shown that the horizontal and vertical transfer matricies for a bending magnet (in the hard-edge approximation) with rotated pole faces are

$$
\mathbf{M}_{H}=\left(\begin{array}{cc}
1 & 0 \\
(\tan \beta) / \rho & 1
\end{array}\right)\left(\begin{array}{cc}
\cos \theta & \rho \sin \theta \\
(-\sin \theta) / \rho & \cos \theta
\end{array}\right)\left(\begin{array}{cc}
1 & 0 \\
(\tan \alpha) / \rho & 1
\end{array}\right),
$$


and

$$
\mathbf{M}_{V}=\left(\begin{array}{cc}
1 & 0 \\
(-\tan \beta) / \rho & 1
\end{array}\right)\left(\begin{array}{cc}
1 & \rho \theta \\
0 & 1
\end{array}\right)\left(\begin{array}{cc}
1 & 0 \\
(-\tan \alpha) / \rho & 1
\end{array}\right)
$$

where $\alpha$ and $\beta$ are the pole face rotation angles at the entrance and exit of the bend as indicated in Figure $4, \rho$ is the radius of curvature of the reference trajectory, and $\theta$ is the bend angle.

Now let $M$ be any two-by-two transfer matrix. Then if $M_{21}$ is not zero we have

$$
\mathbf{M}=\left(\begin{array}{cc}
1 & L_{2} \\
0 & 1
\end{array}\right)\left(\begin{array}{cc}
1 & 0 \\
-1 / f & 1
\end{array}\right)\left(\begin{array}{cc}
1 & L_{1} \\
0 & 1
\end{array}\right)
$$

where

$$
f=-1 / M_{21}, \quad L_{1}=\left(M_{22}-1\right) / M_{21}, \quad L_{2}=\left(M_{11}-1\right) / M_{21} .
$$

Thus $M$ can be expressed as a drift followed by a thin lens followed by another drift. If $\mathbf{M}_{H}$ and $\mathrm{MM}_{V}$ are expressed in this way, then the drift lengths define the principle planes and the thin lens defines the focusing strength of the bend. For the case in which $\alpha=\beta$ one can use (51) and (52) in (54) to determine the $\alpha$ for which the focusing strength of the bend is the same in the horizontal and vertical planes.

\section{Achromatic Bends}

Consider the arrangement of two $\theta^{\circ}$ bends and four quadrupoles shown in Figure 5. The two QF (horizontal focusing) quads are excited with current $I_{1}$ and the two QD (horizontal defocusing) quads are excited with current $I_{2}$. Each bend has radius of curvature $\rho$, and pole faces rotated by angle $\alpha$. The entire arrangement is symmetric about the point half-way between the bends and therefore has (horizontal) transfer matrix

$$
\mathbf{T}=\mathbf{A B A} \sigma \mathbf{C}^{\dagger} \sigma \mathbf{C A B A}
$$

where ABA is the transfer matrix for each bend and $C$ is the transfer matrix from the exit of the first bend to the center of the whole arrangement. 
In order to treat off-momentum particles, the matricies in (55) must include the dispersion, $D$, and it's derivative, $D^{\prime}$. Thus we have

$$
\begin{gathered}
\mathbf{A}=\left(\begin{array}{ccc}
1 & 0 & 0 \\
(\tan \alpha) / \rho & 1 & 0 \\
0 & 0 & 1
\end{array}\right), \quad \mathbf{B}=\left(\begin{array}{ccc}
\cos \theta & \rho \sin \theta & D \\
(-\sin \theta) / \rho & \cos \theta & D^{\prime} \\
0 & 0 & 1
\end{array}\right), \\
\mathbf{C}=\left(\begin{array}{ccc}
C_{11} & C_{12} & 0 \\
C_{21} & C_{22} & 0 \\
0 & 0 & 1
\end{array}\right), \quad \sigma=\left(\begin{array}{lll}
0 & 1 & 0 \\
1 & 0 & 0 \\
0 & 0 & 1
\end{array}\right),
\end{gathered}
$$

where, as shown in reference (1),

$$
D=\rho(1-\cos \theta), \quad D^{\prime}=\sin \theta
$$

Now we can write

$$
\mathbf{B}=\mathbf{L Q L}
$$

where

$$
\begin{gathered}
\mathbf{L}=\left(\begin{array}{ccc}
1 & \lambda & 0 \\
0 & 1 & 0 \\
0 & 0 & 1
\end{array}\right), \quad \mathbf{Q}=\left(\begin{array}{rrr}
1 & 0 & a \\
-q & 1 & b \\
0 & 0 & 1
\end{array}\right), \\
\lambda=\left(B_{11}-1\right) / B_{21}, \quad a=D-\lambda D^{\prime}, \quad b=D^{\prime}, \quad q=-B_{21} .
\end{gathered}
$$

Then we have

$$
\mathbf{T}=\mathbf{A L P L A}, \quad \mathbf{P}=\mathbf{Q N Q}, \quad \mathbf{N}=\sigma \mathbf{R}^{\dagger} \sigma \mathbf{R}, \quad \mathbf{R}=\mathbf{C A L} .
$$

The entire arrangement is achromatic if and only if $P_{13}=P_{23}=0$. Using $(60)$ in (62) we find

$$
P_{13}=N_{12} b=2 b R_{12} R_{22},
$$

and

$$
P_{23}=b\left(N_{22}-q N_{12}+1\right)=2 b\left(R_{11} R_{22}-q R_{12} R_{22}\right) .
$$

Thus the condition for achromaticity becomes

$$
R_{22}=0 \text {. }
$$

This condition imposes only one constraint on the currents $I_{1}$ and $I_{2}$. If we require that $R_{22}=0$ in the vertical plane as well, then $I_{1}$ and $I_{2}$ will be completely specfied. 
We note that in the horizontal plane $\mathbf{R}$ is the transfer matrix from the principle plane near the exit of the first bend to the point half-way between the two bends. The condition, $R_{22}=0$, is then just the condition for point-to-parallel imaging between these two points. (A discussion of point-to-parallel imaging may be found in reference 1.)

\section{HITL Overview}

The design of the HITL is based on beam transport calculations performed by J. D. Larson and T. G. Robinson with Larson's code OPTIC II. These calculations were repeated and checked by $H$. N. Brown with the TRANSPORT code.

Figure 6 is a schematic diagram of the HITL showing the various sections into which the line is divided. Sections $11-23$ contain 37 quadrupoles and three pairs of horizontal bending magnets as indicated in the figure. The quadrupoles have a steel length of 6.0 inches and an effective length of 8.0 inches. The magnetic field gradient in each quadrupole is $0.25 \mathrm{~T} / \mathrm{m}$ per amp. The pole-faces of each bending magnet are rotated to provide equal focusing strengths in the horizontal and vertical planes (as discussed in section 2), and each pair of bending magnets forms a bending section which is achromatic. The radius of curvature is 60 inches in the $90^{\circ}$ bends and 30 inches in the $24^{\circ}$ and $69^{\circ}$ bends.

After acceleration in Tandem van de Graaf MP7, the heavy ion beam is focused by a quadrupole doublet and analyzed by the first $90^{\circ}$ bending magnet. Image slits located between the two $90^{\circ}$ bends allow for energy stabilization of MP7 and form the object point of the second $90^{\circ}$ bend. The final stripping of the ions occurs in a carbon foil located between the two bends. Any ions left with unwanted charge states after the final stripping are swept out of the beam by the second $90^{\circ}$ bend. The two horizontal focusing quadrupoles between the two bends are adjusted to make the total $180^{\circ}$ bend achromatic. The four quadrupoles just downstream of the second $90^{\circ}$ bend serve as a matching section which matches the parameters of the beam ellipse emerging from the bend to those required for transport through the rest of the transfer line. In the remaining sections (13-23) of the line the beam ellipse is transported through two $24^{\circ}$ bends, two $69^{\circ}$ bends, and is then matched to the AGS lattice. The details of this transport are discussed in the following sections 
where the results of beam transport calculations are presented. These calculations treat the quadrupoles and dipoles in the hard-edge approximation and have been carried out for the case in which the particle momentum is $0.225 \mathrm{GeV} / \mathrm{c}$ per proton charge. (It should be noted that the calculations assume the transfer line lies in a plane. The line does in fact have a downward pitch of 3.3 milliradians between the second $24^{\circ}$ bend and the first $69^{\circ}$ bend, and has a downward pitch of $5.4^{\circ}$ between the two $69^{\circ}$ bends. This results in a small coupling between the horizontal and vertical planes which is not included in the calculations.)

\section{The HITL Cell (Sections 13 and 14)}

HITL Sections 13 and 14 contain four quadrupoles-13QH1, 13QV1, $14 \mathrm{QV1}$, and 14QH1-which are positioned to form the symmetric doublet cell described in section 1.8. (QH and QV refer to Horizontal and Vertical focusing quadrupoles respectively.) The cell runs from profile monitor 12MW165 $(s=0)$ to 14MW240 $(s=2(d+2 l))$ as indicated in Figure 7 . The quads are all excited with the same current, $I$, and $d=18$ inches, $l=1438$ inches.

One can make best use of the available aperture in the cell if the matching section upsteam of section 13 is tuned so that the beam ellipse enters the cell (at 12MW165) with parameters $\alpha=0$ and $\beta=\beta_{0}$ given by equation (49). As discussed in section 1.8, the cell will then image a waist to a waist, and the beam ellipse will emerge from the cell (at 14MW240) with the same parameters it had at 12MW165. For each current, $I$, one can compute $\beta_{0}(I)$, which can then be used with $\alpha=0$ in equation (14) to obtain $\beta(s)$. In the horizontal plane $\beta(s)$ reaches it's maximum value, $\beta_{\max }(I)$, in the QH quads. In the vertical plane the same maximum is reached in the QV quads.

Figures 8 and 9 are plots of $\beta_{\max }(I)$ which show that waist-to-waist imaging is only possible for currents in the regions $0<I<3.69$ and $4.16<I<5.57$ (amps). As one approaches the end-points of these regions $\beta_{\max }$ becomes infinite. In the region between 0 and 3.69 amps, $\beta_{\max }(I)$ reaches a minimum value of 89 meters. A slightly larger minimum (96 meters) is reached in the region between 4.16 and 5.57 amps.

Historically the HITL cell has been operated at a current of about $\mathbf{5 . 0}$ amps (when the particle momentum is $0.225 \mathrm{GeV} / \mathrm{c}$ per proton charge). 
At this current $\beta_{0}=13.8$ meters in the horizontal plane and 22.9 meters in the vertical plane, $\beta_{\max }=110$ meters, and the phase advance $\psi=255^{\circ}$. Figures 10-14 show the behavior of $\beta_{\max }(I), \beta_{0}(I)$, and the phase advance near $I=5.0$ amps. Figures 15 and 16 are plots of $\beta(s)$ at this current.

If the HITL cell is operated at a higher current, say $I=5.52 \mathrm{amps}$, then $\beta_{0}=3.8$ meters in the horizontal plane and 5.4 meters in the vertical plane, $\beta_{\max }=350$ meters, and the phase advance $\psi=331^{\circ}$. Figures 17 and 18 are plots of $\beta(s)$ at this current.

It is interesting to compare the HITL symmetric doublet cell with a FODO cell (described in section 1.9) constructed of quadrupoles with the same strength per amp. If we let $L=l+d / 2$ in Figure 3 and compute $\beta_{\max }(I)$ using equation (50), we find that $\beta_{\max }$ reaches it's smallest value (122 meters) when $I=0.5$ amps. Thus the FODO cell has approximately the same acceptance as the HITL cell but requires only one tenth as much current. Figure 19 is a plot of $\beta(s)$ for the FODO cell with $I=0.5$ amps.

\section{The $24^{\circ}$ Bends (Sections 15-19)}

The beam ellipse emerges from the HITL cell and enters section 15 at profile monitor 14MW240 with parameters $\alpha=0$ and $\beta=\beta_{0}(I)$. It is then transported through sections 15-19 which contain 12 quadrupoles and two $24^{\circ}$ bends as indicated in Figure 20. Here $L_{1}=1438, L_{2}=1138, L_{3}=665$, $L_{b}=289, L_{2}^{\prime}=1118, L_{1}^{\prime}=1488, d_{1}=18$, and $d_{2}=29$ (all in inches). The pole faces at the entrance and exit of each bend are rotated by $7.09^{\circ}$ to achieve equal focusing strengths in the horizontal and vertical planes, and the currents in quadrupoles $17 \mathrm{QH} 1,17 \mathrm{QV} 1,17 \mathrm{QV} 2$, and $17 \mathrm{QH} 2$ are adjusted so that the two $24^{\circ}$ bends are achromatic as discussed in section 3. These currents are found (by computation) to be $I_{1}=8.50$ amps and $I_{2}=8.05$ amps (when the particle momentum per proton charge is 0.225 $\mathrm{GeV} / \mathrm{c}$ ), where $I_{1}$ is the current in $17 \mathrm{QH} 1$ and $17 \mathrm{QH} 2$, and $I_{2}$ is the current in $17 \mathrm{QV} 1$ and $17 \mathrm{QV} 2$. The currents in quadrupoles $15 \mathrm{QH} 1$, 15QV1, 16QV1, 16QH1, 18QH1, 18QV1, 19QV1, and 19QH1 are adjusted so that the beam ellipse leaves section 19 (at profile monitor 19MW250) with the same parameters it had at 14MW240. Since the placement of elements in sections $15-19$ is approximately symmetric about the point half-way between the two $24^{\circ}$ bends, one can tune the quadrupole currents so that the ellipse parameter $\beta(s)$ is symmetric about this point. The 
currents in 19QH1, 19QV1, 18QV1, and 18QH1 should then approximately equal the currents in 15QH1, 15QV1, 16QV1, and 16QH1 respectively.

The quadrupole currents required to insure that the beam ellipse has the same parameters (i.e. $\alpha=0$ and $\beta=\beta_{0}(I)$ ) at 14MW240 and 19MW250 are not unique. One set of currents which meets this requirement for the case in which the HITL cell current is $\mathbf{5 . 0}$ amps is listed in Table I.

\begin{tabular}{|c|c|c|c|}
\hline \multicolumn{4}{|c|}{ Table I: Quad currents (amps). } \\
(HITL cell $I=5.0$ amps) \\
\hline Quad & Current & Quad & Current \\
\hline 15QH1 & 5.16 & $19 Q H 1$ & 5.16 \\
15QV1 & 5.17 & $19 Q V 1$ & 5.17 \\
16QV1 & 7.16 & $18 Q V 1$ & 7.14 \\
16QH1 & 7.17 & $18 Q H 1$ & 7.15 \\
\hline
\end{tabular}

Figures 21-24 are the corresponding plots of $\beta(s)$. Another set of currents is listed in Table II and Figures 25-28 are the corresponding plots of $\beta(s)$.

\begin{tabular}{|c|c|c|c|}
\hline \multicolumn{4}{|c|}{$\begin{array}{c}\text { Table II: Quad currents (amps). } \\
\text { (HITL cell } I=5.0 \text { amps) }\end{array}$} \\
\hline Quad & Current & Quad & Current \\
\hline 15QH1 & 5.00 & $19 Q H 1$ & 5.00 \\
15QV1 & 5.05 & $19 Q V 1$ & 5.05 \\
16QV1 & 7.17 & $18 Q V 1$ & 7.14 \\
16QH1 & 7.17 & $18 Q H 1$ & 7.14 \\
\hline
\end{tabular}

For the case in which the HITL cell current is increased to 5.52 amps a possible set of currents is listed in Table III and Figures 29-32 are the corresponding plots of $\beta(s)$.

\begin{tabular}{|c|c|c|c|}
\hline \multicolumn{4}{|c|}{$\begin{array}{c}\text { Table III: Quad currents (amps). } \\
\text { (HITL cell } I=5.52 \text { amps) }\end{array}$} \\
\hline Quad & Current & Quad & Current \\
\hline 15QH1 & 5.40 & $19 Q H 1$ & 5.40 \\
15QV1 & 5.40 & $19 Q V 1$ & 5.40 \\
16QV1 & 7.35 & $18 Q V 1$ & 7.32 \\
16QH1 & 7.41 & $18 Q H 1$ & 7.38 \\
\hline
\end{tabular}

(All currents have been obtained for the case in which the particle momentum per proton charge is $0.225 \mathrm{GeV} / \mathrm{c}$.) 


\section{The $69^{\circ}$ Bends (Sections 20-22)}

The beam ellipse enters section 20 at profile monitor $19 \mathrm{MW} 250$ with parameters $\alpha=0$ and $\beta=\beta_{0}(I)$, and is transported through sections 20-22 which contain eight quadrupoles and two $69^{\circ}$ bends as indicated in Figure 33. Here $L_{1}=1488, L_{2}=1118, L_{3}=639, L_{b}=585, d_{1}=18$, $d_{2}=40$, and $d_{3}=27$ (all in inches). The pole faces at the entrance and exit of each bend are rotated by $20.26^{\circ}$ to achieve equal focusing strengths in the horizontal and vertical planes, and the currents in quadrupoles $22 \mathrm{QH} 1,22 \mathrm{QV} 1,22 \mathrm{QV} 2$, and $22 \mathrm{QH} 2$ are adjusted so that the two bends are achromatic as discussed in section 3 . The currents required for achromaticity are found (by computation) to be $I_{1}=6.15$ amps and $I_{2}=5.98 \mathrm{amps}$ (when the particle momentum per proton charge is $\mathbf{0 . 2 2 5}$ $\mathrm{GeV} / \mathrm{c}$ ), where $I_{1}$ is the current in $22 \mathrm{QH} 1$ and $22 \mathrm{QH} 2$, and $I_{2}$ is the current in 22QV1 and 22QV2.

The transport of the beam ellipse through sections 20-22 is dominated by the strong focusing effect of the $69^{\circ}$ bends. One finds that in order to keep beta from becoming too large between the bends, it must be made sufficiently large in quadrupoles $21 \mathrm{QV} 1$ and $21 \mathrm{QH} 1$. The currents in quadrupoles 20QH1, 20QV1, 21QV1, 21QH1 must therefore be adjusted so that an optimum condition is reached in which beta is as small as possible in both locations. One proceedes by first adjusting the currents in $20 \mathrm{QH1}$ and $20 \mathrm{QV} 1$ so that the maximum beta in $21 \mathrm{QV} 1$ and $21 \mathrm{QH} 1$ is the same in the horizontal and vertical planes. Then the currents in 21QV1 and $21 \mathrm{QH} 1$ are adjusted so that the maximum beta between the two bends is the same in the horizontal and vertical planes. If the maximum beta between the bends is larger (smaller) than the maximum beta in $21 \mathrm{QV1}$ and $21 \mathrm{QH} 1$, then the currents in $20 \mathrm{QH1}$ and $20 \mathrm{QV} 1$ must be increased (decreased) until the maximum beta is the same in both locations. Table IV lists the quadrupole currents required to achieve this optimum condition for the case in which the HITL cell current $I=5.0$ amps (and $\alpha=0, \beta=\beta_{0}(I)$ at 19MW250). Figures 34-37 are the corresponding plots of $\beta(s)$. At profile monitor 22MW115 $\alpha=-1.27, \beta=0.97$ (meters) in the horizontal plane, and $\alpha=-1.33, \beta=0.89$ in the vertical plane. 


\begin{tabular}{|c|c|}
\hline $\begin{array}{c}\text { Table IV: Quad currents (amps). } \\
\text { (HITL cell } I=5.0 \text { amps) }\end{array}$ \\
\hline Quad & Current \\
\hline 20QH1 & 5.62 \\
20QV1 & 5.64 \\
21QV1 & 6.84 \\
$21 Q H 1$ & 6.91 \\
\hline
\end{tabular}

For the case in which the HITL cell current is 5.52 amps the quadrupole currents are listed in Table $\mathrm{V}$ and Figures 38-41 are the corresponding plots of $\beta(s)$. At profile monitor 22MW115 $\alpha=-1.20, \beta=0.92$ (meters) in the horizontal plane, and $\alpha=-1.32, \beta=0.85$ in the vertical plane.

\begin{tabular}{|c|c|}
\hline \multicolumn{2}{|c|}{$\begin{array}{c}\text { Table V: Quad currents (amps). } \\
\text { (HITL cell } I=5.52 \text { amps) }\end{array}$} \\
\hline Quad & Current \\
\hline 20QH1 & 5.47 \\
20QV1 & 5.48 \\
21QV1 & 7.21 \\
21QH1 & 7.27 \\
\hline
\end{tabular}

(All currents have been obtained for the case in which the particle momentum per proton charge is $0.225 \mathrm{GeV} / \mathrm{c}$.)

\section{Matching to the AGS (Section 23)}

The beam ellipse enters section 23 at profile monitor 22MW115 with the parameters given in the previous section. Section 23 contains seven quadrupoles and an electrostatic inflector as indicated in Figure 42 . Here $L_{1}=102, L_{2}=82, L_{3}=39, L_{4}=100, d_{1}=18, d_{2}=22$ (all in inches), and $L_{I}=2.0$ (meters). The quadrupoles are tuned to match the beam ellipse parameters to the AGS lattice parameters at the exit of the inflector. They are excited as two triplets with currents $I_{1}, I_{2}, I_{3}$, and $I_{4}$ as shown in Table VI. 


\begin{tabular}{|c|c|c|c|}
\hline \multicolumn{4}{|c|}{ Table VI: Section 23 Quad Excitation } \\
\hline Quad & Current & Quad & Current \\
\hline $23 Q V 1$ & $I_{1}$ & $23 Q V 2$ & $I_{3}$ \\
$23 Q H 1$ & $I_{2}$ & $23 Q H 3$ & $I_{4}$ \\
$23 Q H 2$ & $I_{2}$ & $23 Q V 4$ & $I_{3}$ \\
23QV2 & $I_{1}$ & & \\
\hline
\end{tabular}

The desired match can be achieved in the following way. Let $\alpha_{x}, \beta_{x}, \alpha_{y}, \beta_{y}$ be the beam ellipse parameters at the exit of the inflector, and let $\alpha_{x 0}, \beta_{x 0}$, $\alpha_{y 0}, \beta_{y 0}$ be the AGS lattice parameters at this location. (Subscripts $x$ and $y$ refer to the horizontal and vertical planes, and $\alpha_{x 0}=-1.658$, $\beta_{x 0}=19.022$ meters, $\alpha_{y 0}=1.247, \beta_{y 0}=13.063$ meters.) The beam ellipse parameters at the inflector exit depend on the four currents, $I_{j}$, and if we define

$$
\sigma^{2}=\left(\frac{\alpha_{x}-\alpha_{x 0}}{\alpha_{x 0}}\right)^{2}+\left(\frac{\beta_{x}-\beta_{x 0}}{\beta_{x 0}}\right)^{2}+\left(\frac{\alpha_{y}-\alpha_{y 0}}{\alpha_{y 0}}\right)^{2}+\left(\frac{\beta_{y}-\beta_{y 0}}{\beta_{y 0}}\right)^{2},
$$

they will be matched to the AGS lattice whenever

$$
\sigma\left(I_{1}, I_{2}, I_{3}, I_{4}\right)=0 .
$$

For any set of currents, $\sigma\left(I_{1}, I_{2}, I_{3}, I_{4}\right)$ is a measure of how well the beam ellipse parameters are matched to the AGS lattice. The closer $\sigma$ is to zero the better the match. For a given set of ellipse parameters at 22MW115, a simple grid search of the four-dimensional space of currents locates the currents for which equation (67) is satisfied. For the case in which the HITL cell current is 5.0 amps (and the beam ellipse is transported to 22MW115 as discussed in the previous sections) one finds $I_{1}=17.3$, $I_{2}=13.3, I_{3}=10.3$, and $I_{4}=13.2$ (amps). The corresponding plots of $\beta(s)$ in section 23 are shown in Figures 43 and 44 . If the HITL cell current is increased to 5.52 amps one finds $I_{1}=17.2, I_{2}=13.25, I_{3}=10.7$, and $I_{4}=13.9$ (amps). The corresponding plots of $\beta(s)$ are shown in Figures 45 and 46. (All currents have been obtained for the case in which the particle momentum per proton charge is $0.225 \mathrm{GeV} / \mathrm{c}$.)

One can determine the sensitivity of the match-i.e. the sensitivity of $\sigma$-to the currents by varying each current in turn while keeping the other currents fixed. If the currents are varied about the point for which $\sigma=0$, one finds that $\sigma$ will increase from zero to one when the variations in $I_{1}$, $I_{2}, I_{3}$, and $I_{4}$ are $0.1,0.05,0.5$, and 0.7 (amps) respectively. The match is therefore most sensitive to $I_{1}$ and $I_{2}$ and least sensitive to $I_{3}$ and $I_{4}$. 


\section{References}

1. D. C. Carey, The Optics of Charged Particle Beams, Harwood Academic Publishers, New York, 1987.

2. K. L. Brown, F. Rothacker, D. C. Carey, Ch. Iselin, 'Transport, A Computer Program for Designing Charged Particle Beam Transport Systems', CERN 80-04, Super Proton Synchrotron Division, 18 March 1980. 


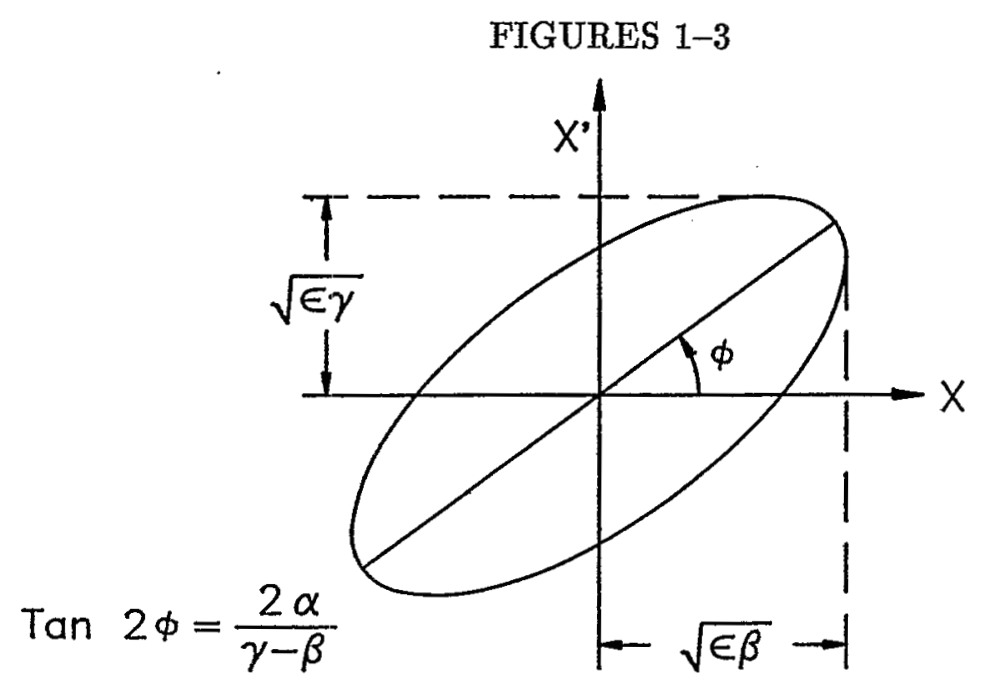

Figure 1. The beam ellipse.

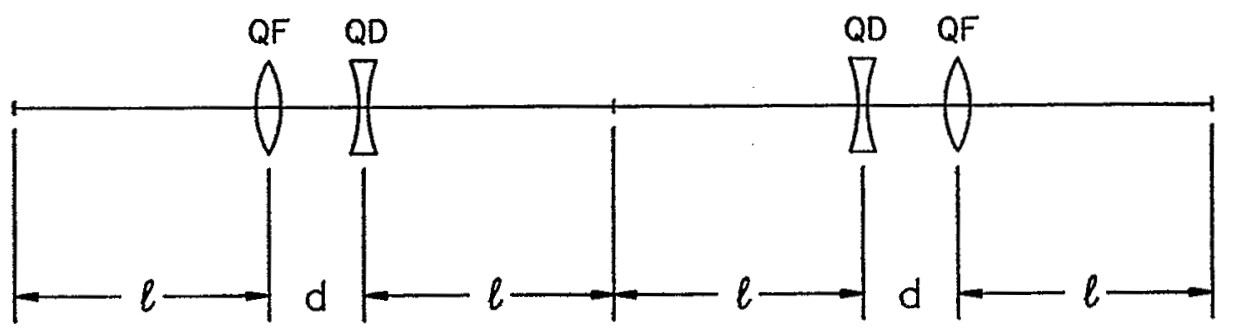

Figure 2. The symmetric doublet cell.

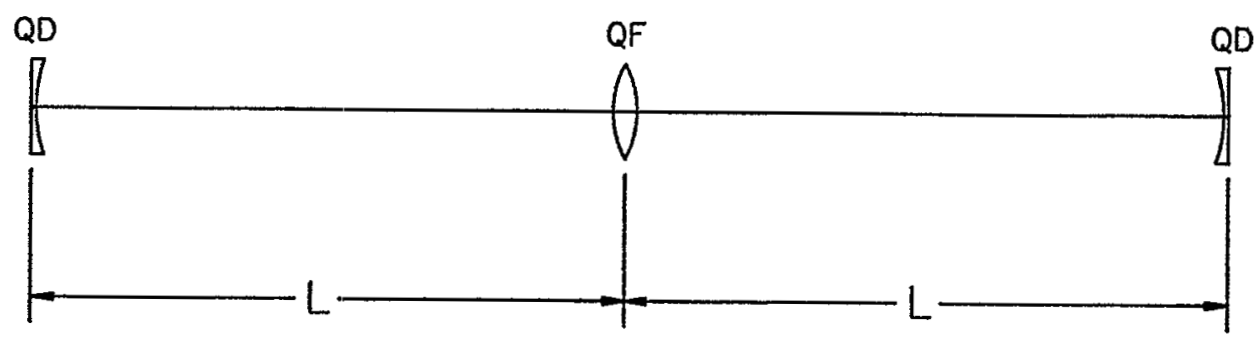

Figure 3. The FODO cell. 


\section{FIGURES 4-5}

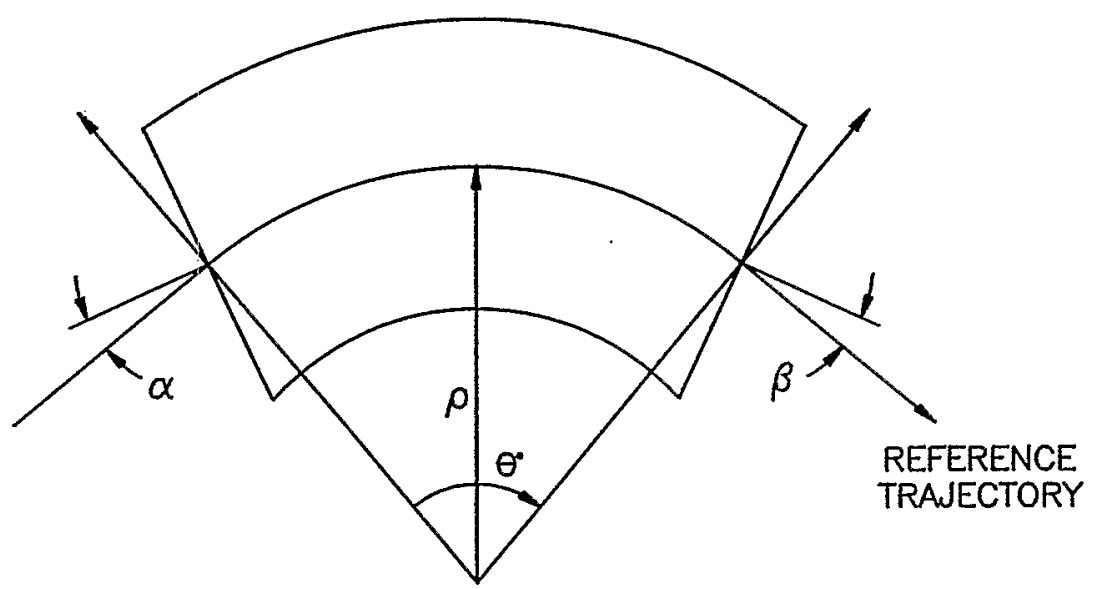

Figure 4. Bending magnet with pole-face rotation.

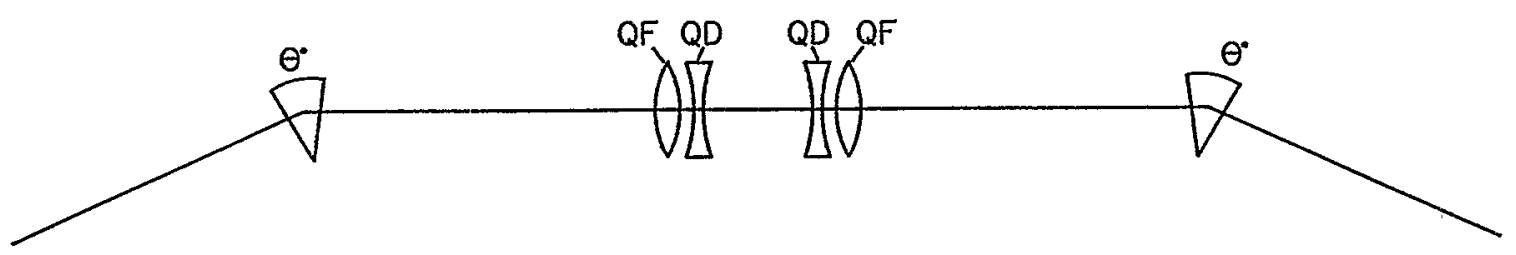

Figure 5. Achromatic bends. 
FIGURE 6

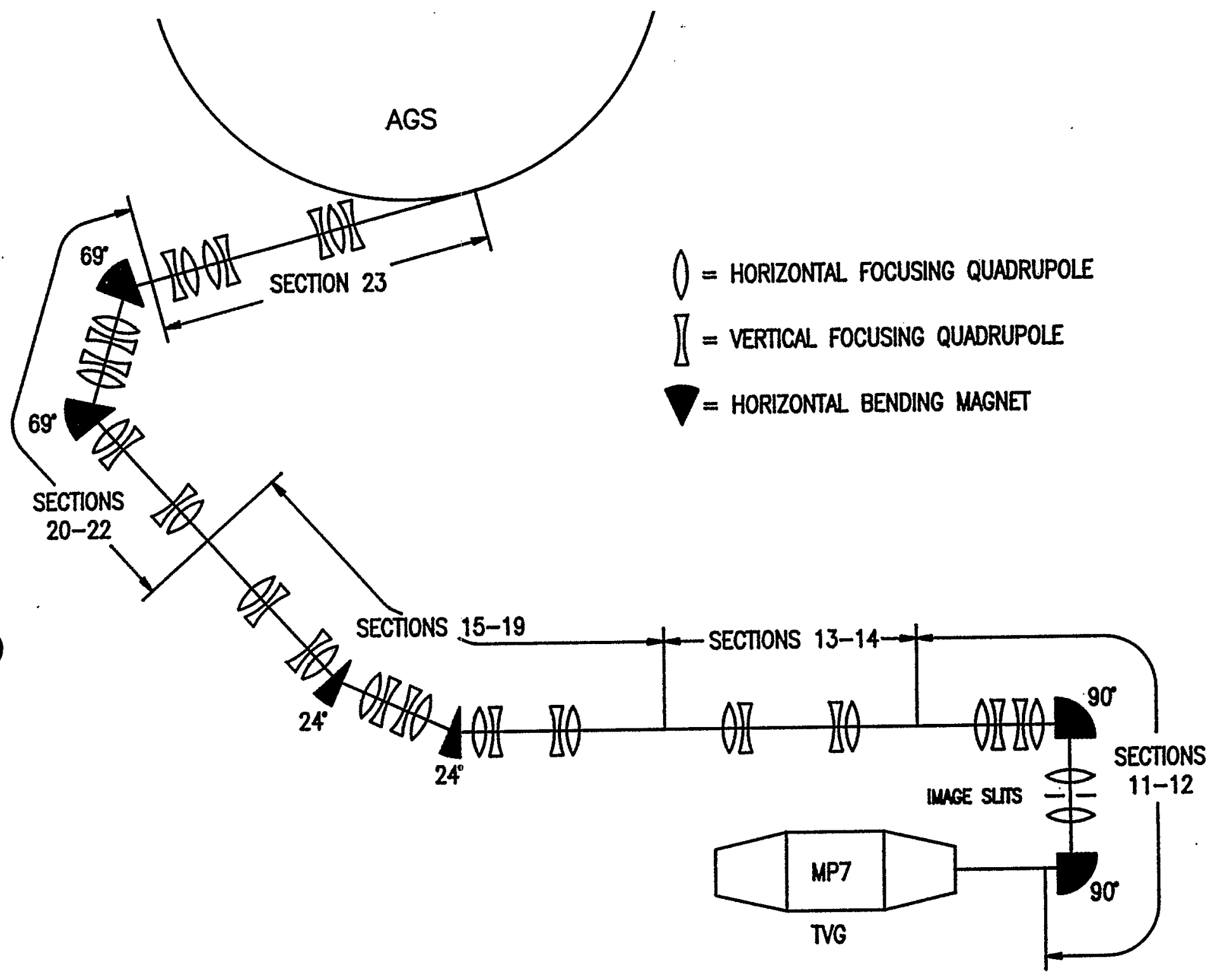

Figure 6. The Heavy Ion Transfer Line. 
FIGURES $7-9$

\section{HITL SECTIONS $13-14$ \\ BEAM DIRECTION}

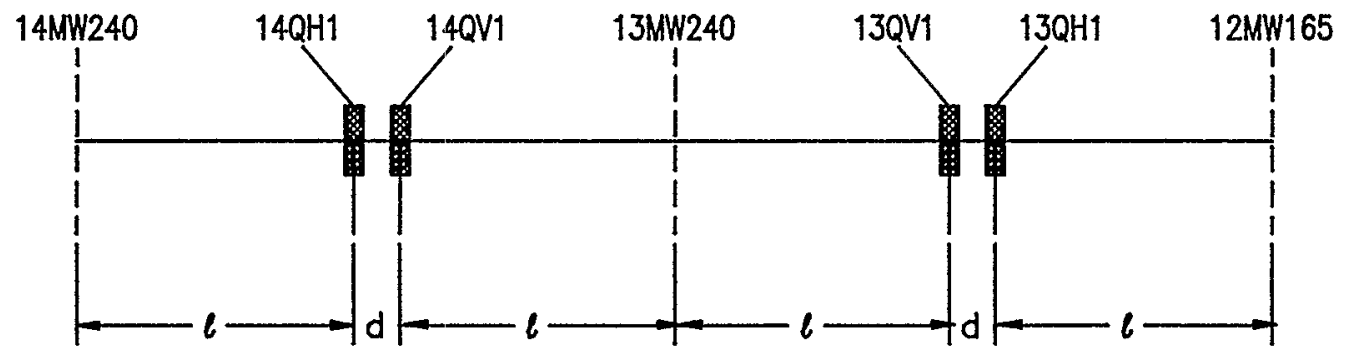

$i=$ MULTIWIRE PROFILE MONITOR

Figure 7. The HITL cell.
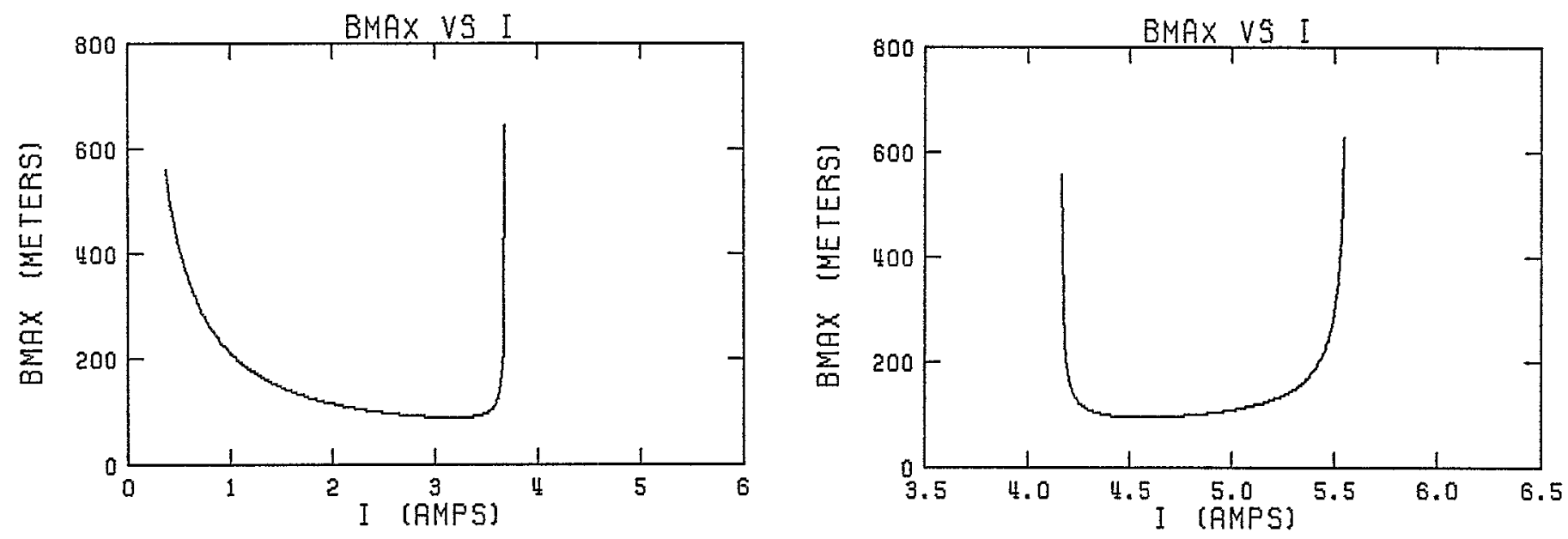

Figures 8 and 9: HITL cell $\beta_{\max }$ vs $I$. 
FIGURES 10-14 (HITL Cell)

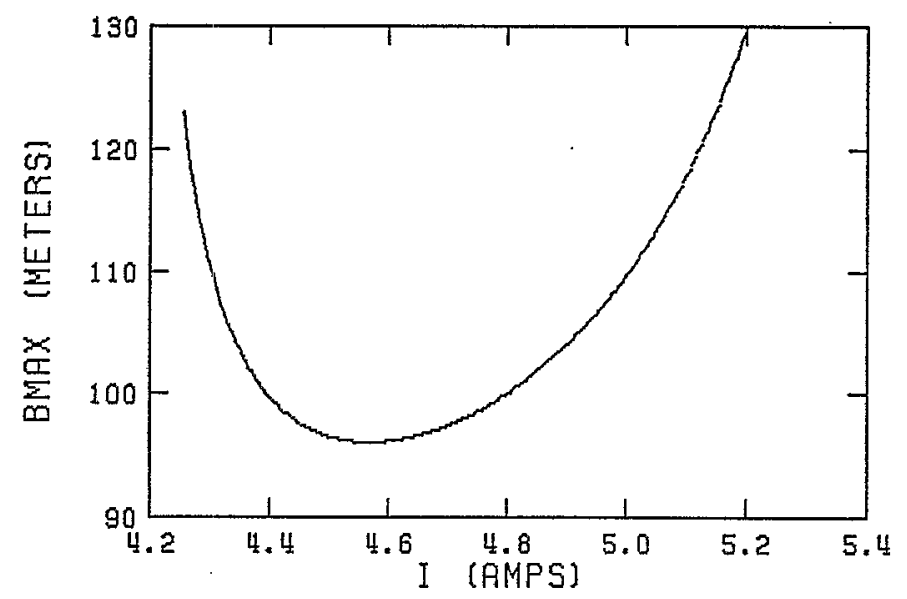

Figure 10. $\beta_{\max }$ vs $I$.
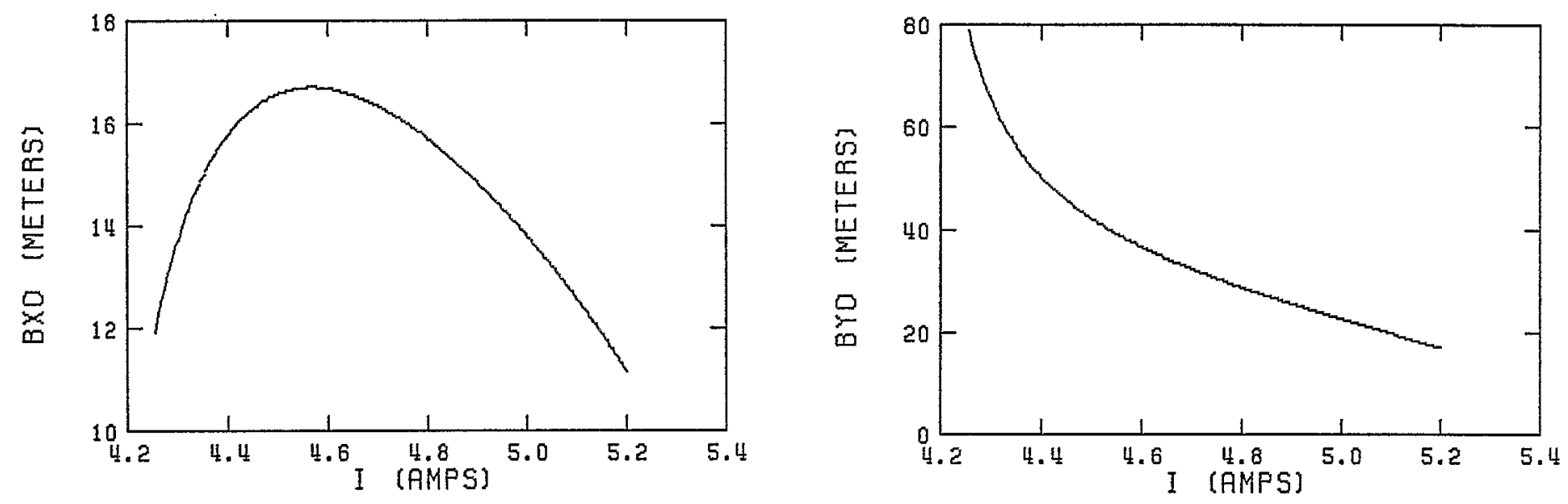

Figures 11 and 12: $\operatorname{Horz}(\mathrm{x})$ and $\operatorname{Vert}(\mathrm{y}) \beta_{0}(I)$.
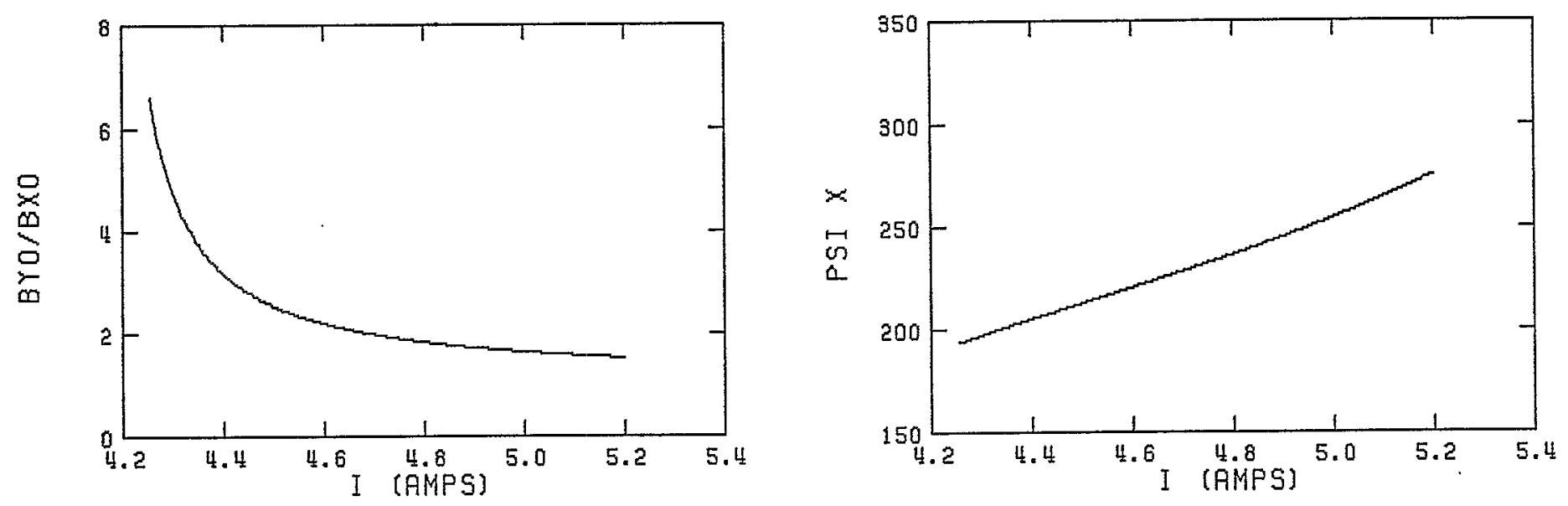

Figures 13 and 14: $\beta_{y 0} / \beta_{x 0}$ vs $I$, and phase advance $\psi(I)$. 
FIGURES 15-19 (HITL and FODO Cells)
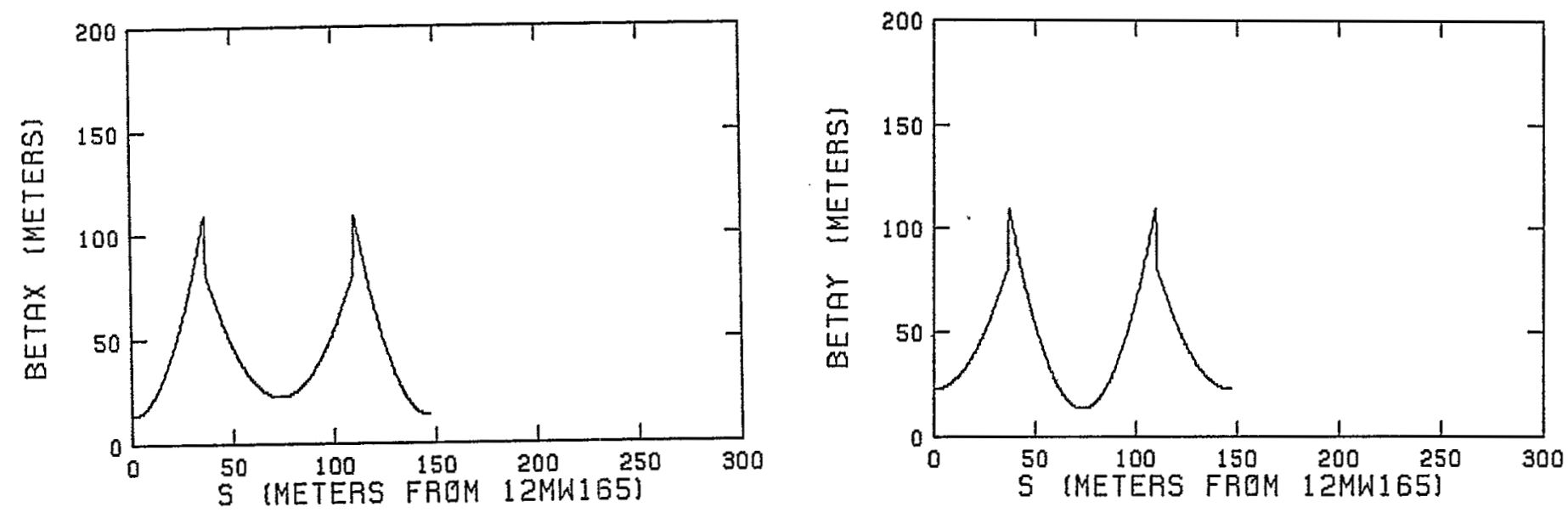

Figures 15 and 16: Horz and Vert $\beta(s), I=5.0$.
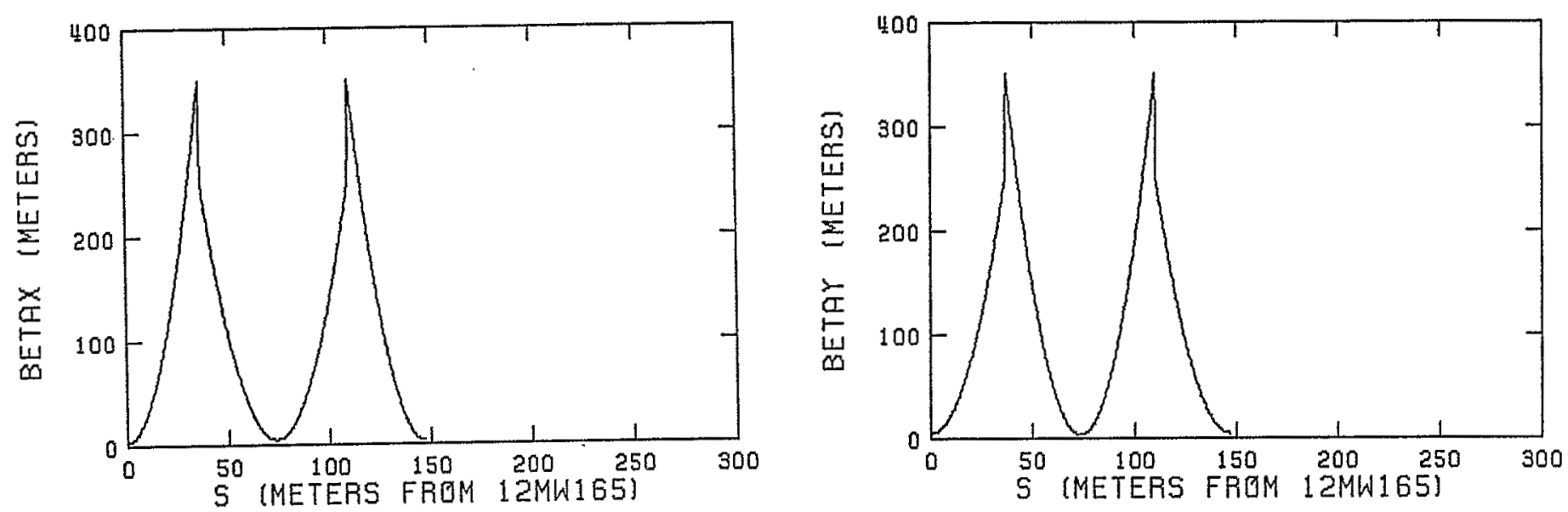

Figures 17 and 18: Horz and Vert $\beta(s), I=5.52$.

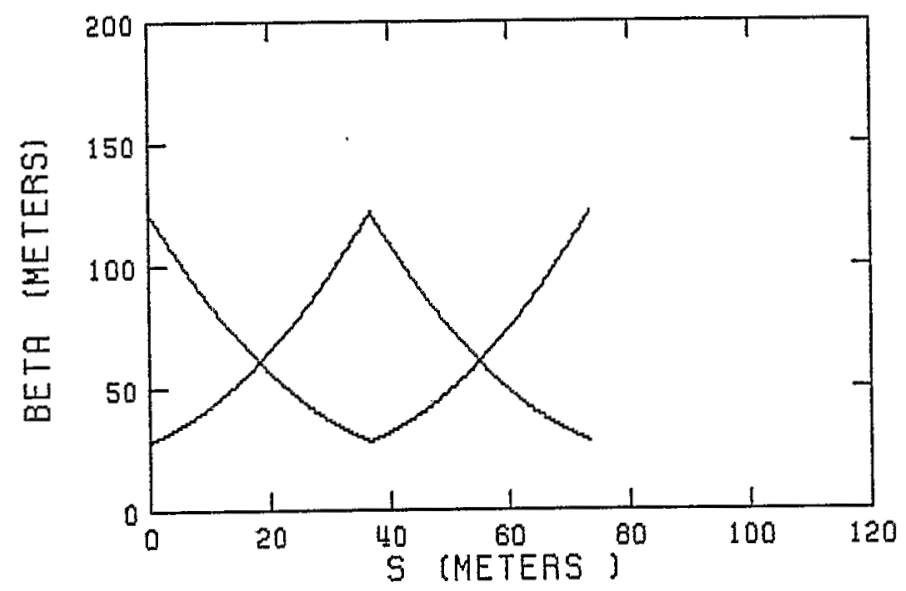

Figure 19. FODO cell $\beta(s), I=0.5$. 
FIGURE 20

\section{HITL SECTIONS $15-19$}

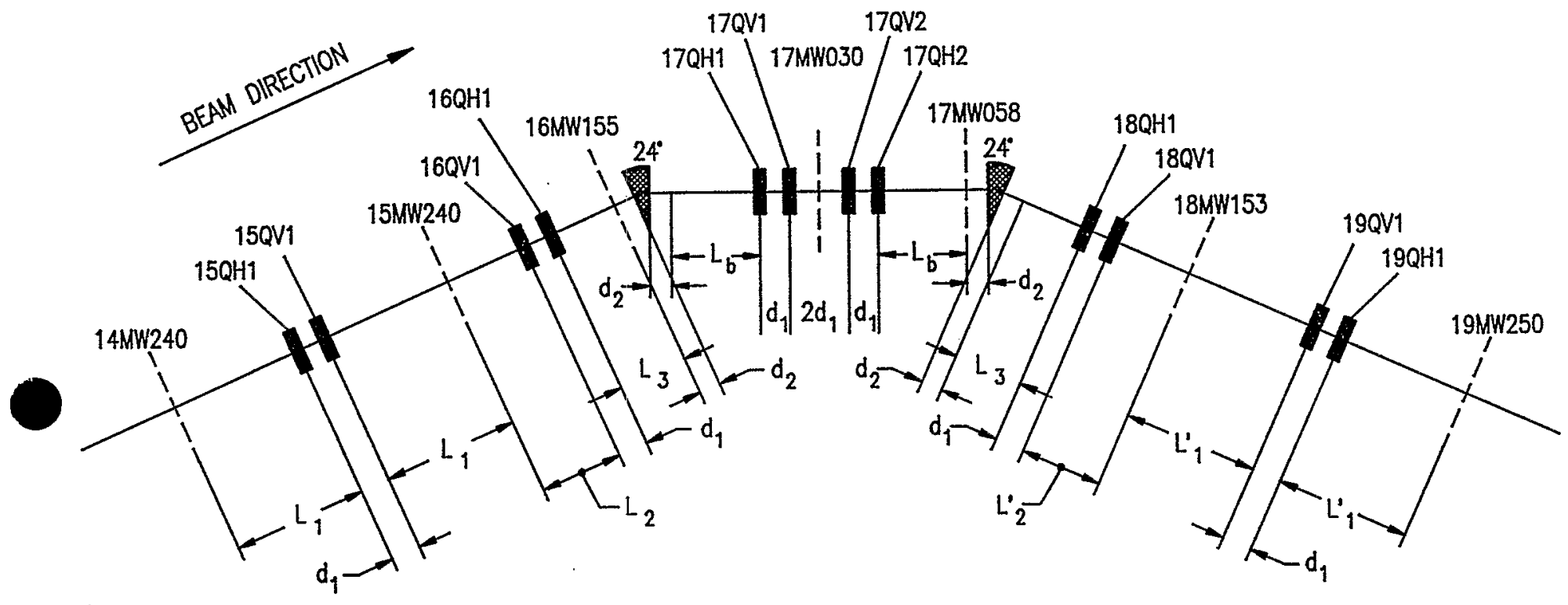

Figure 20. HITL sections 15-19. 
FIGURES 21-24 (HITL Sections 15-19)
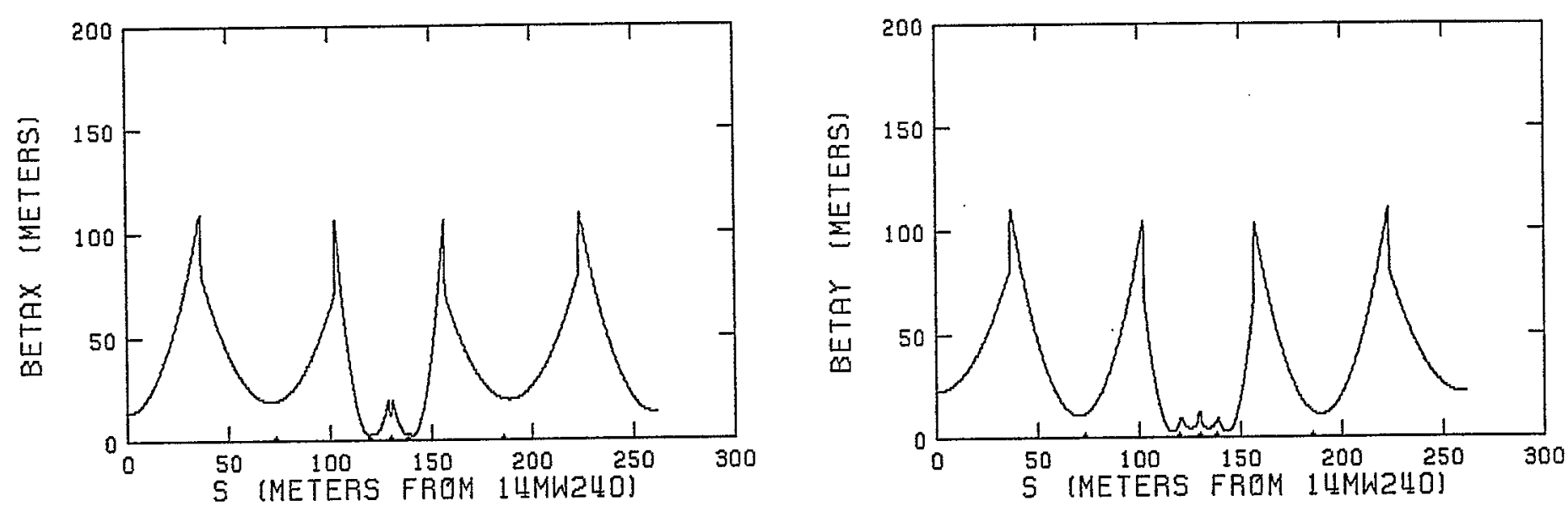

Figures 21 and 22: Horz and Vert $\beta(s), I=5.0$.
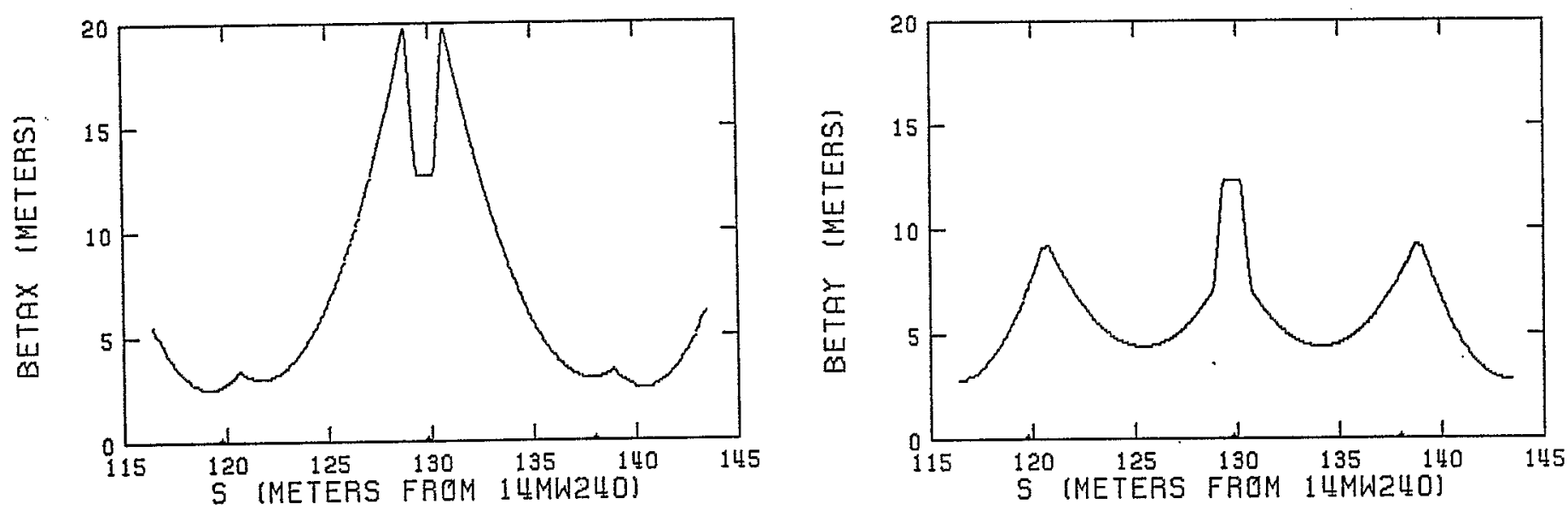

Figures 23 and 24: Horz and Vert $\beta(s)$ in bend region, $I=5.0$. 
FIGURES 25-28 (HITL Sections 15-19)
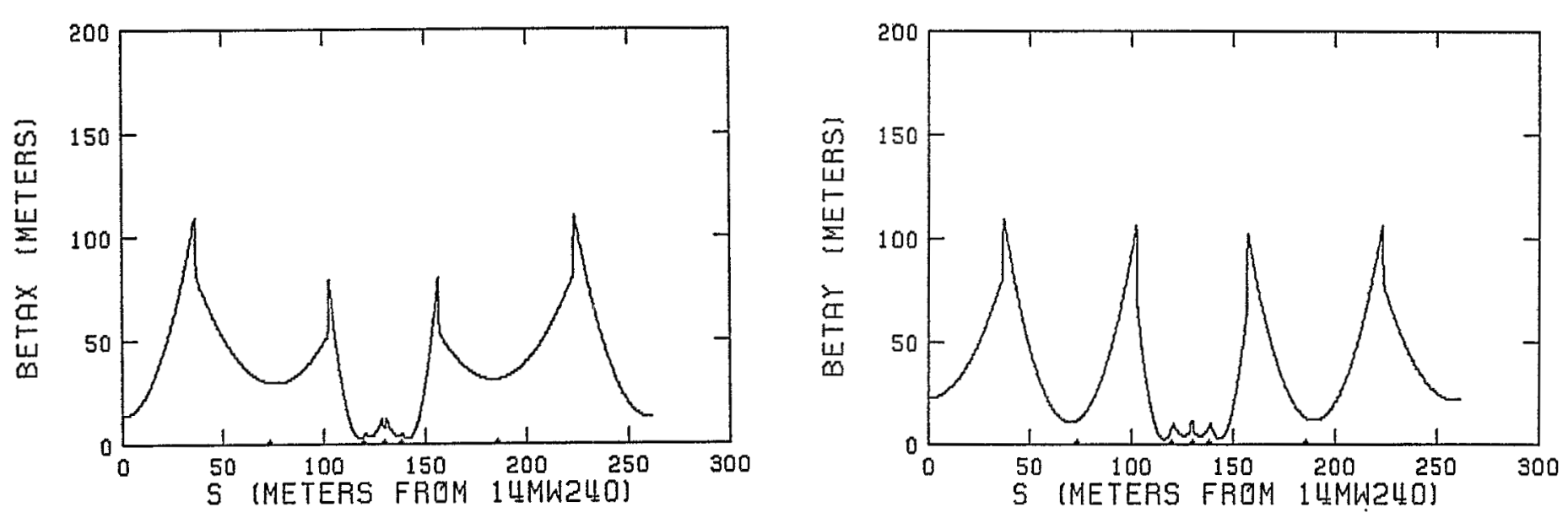

Figures 25 and 26: Horz and Vert $\beta(s), I=5.0$.
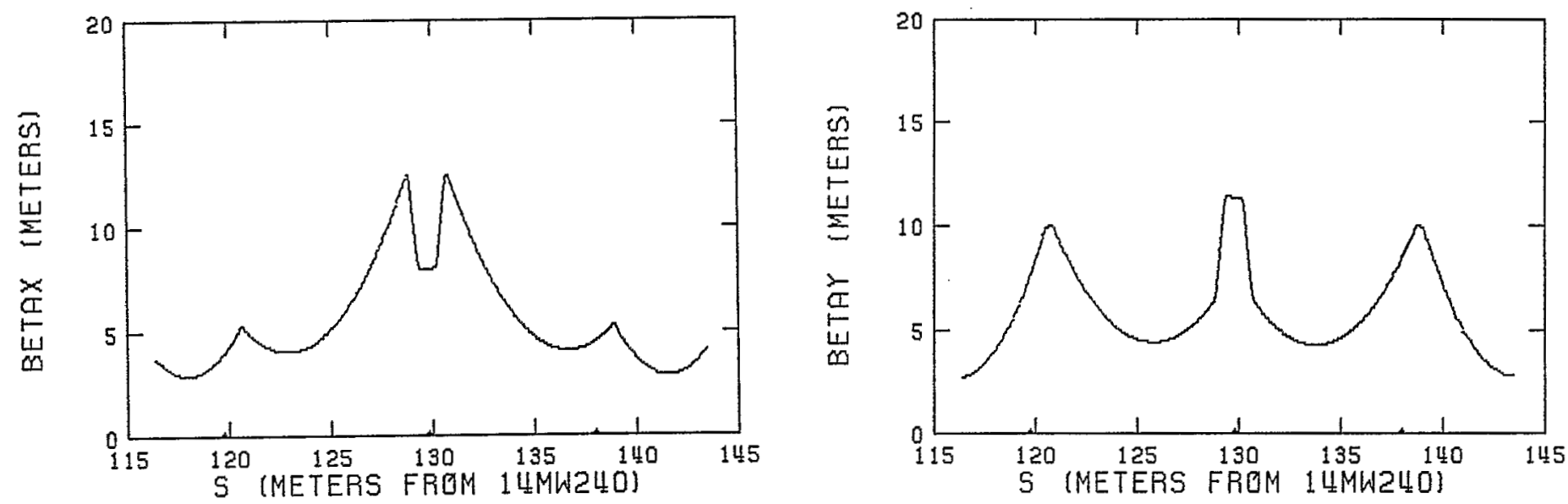

Figures 27 and 28: Horz and Vert $\beta(s)$ in bend region, $I=5.0$. 
FIGURES 29-32 (HITL Sections 15-19)
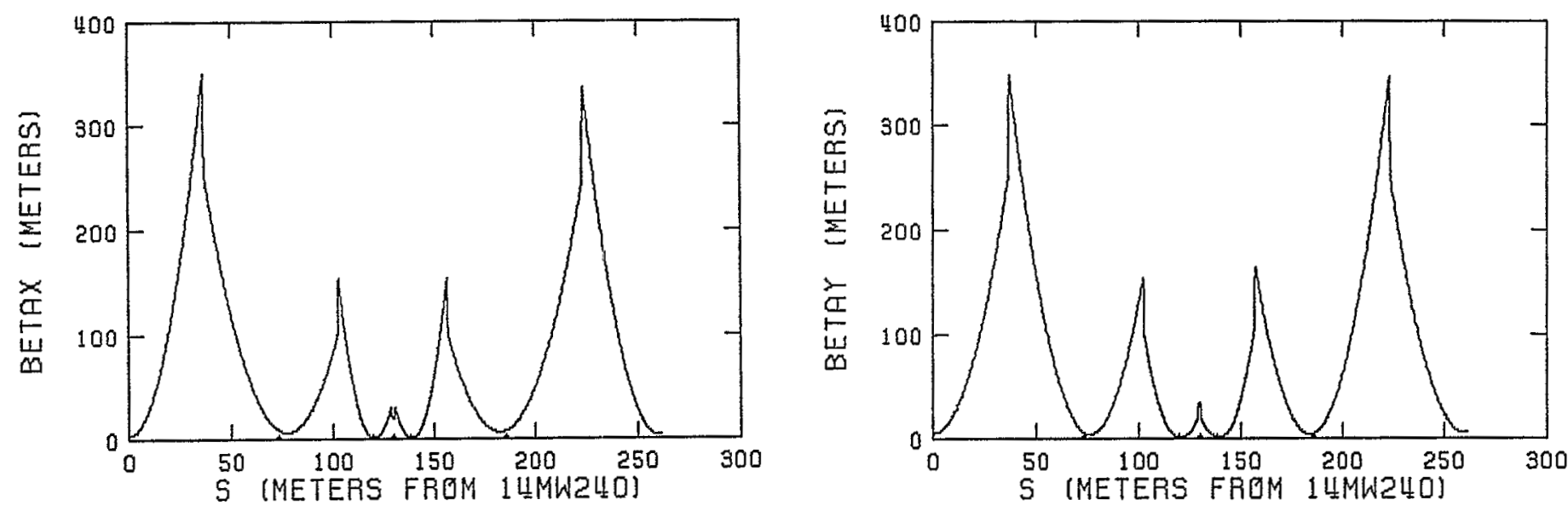

Figures 29 and 30: Horz and Vert $\beta(s), I=5.52$.
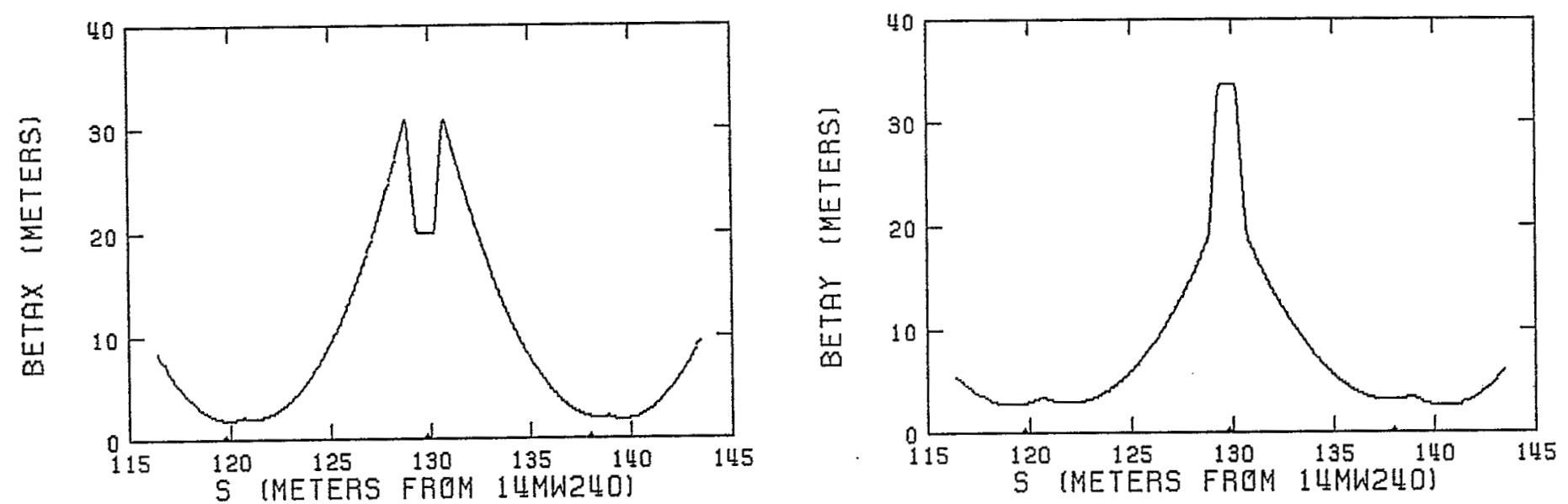

Figures 31 and 32: Horz and Vert $\beta(s)$ in bend region, $I=5.52$. 
HITL SECTIONS $20-22$

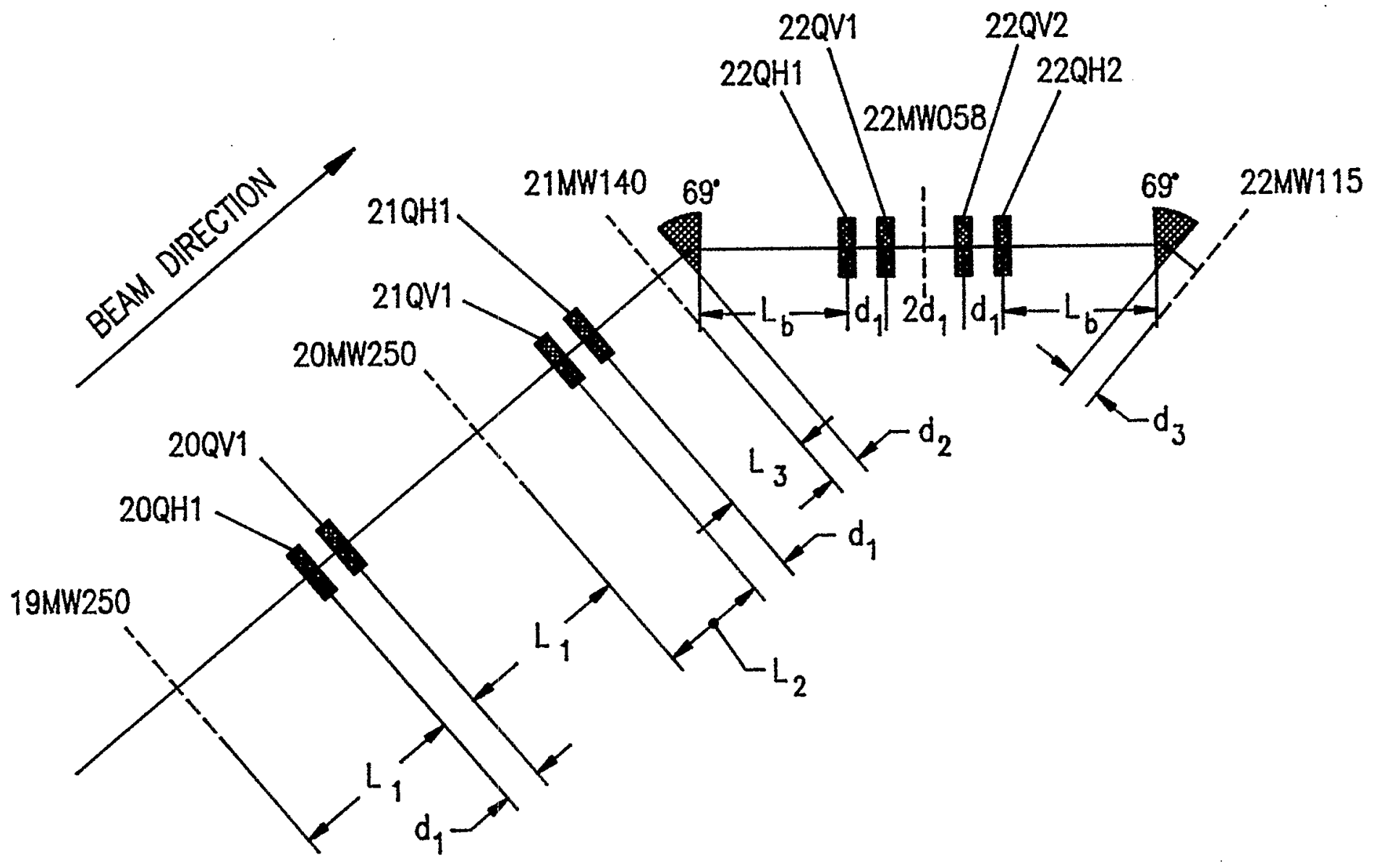

Figure 33. HITL sections 20-22. 
FIGURES 34-37 (HITL Sections 20-22)
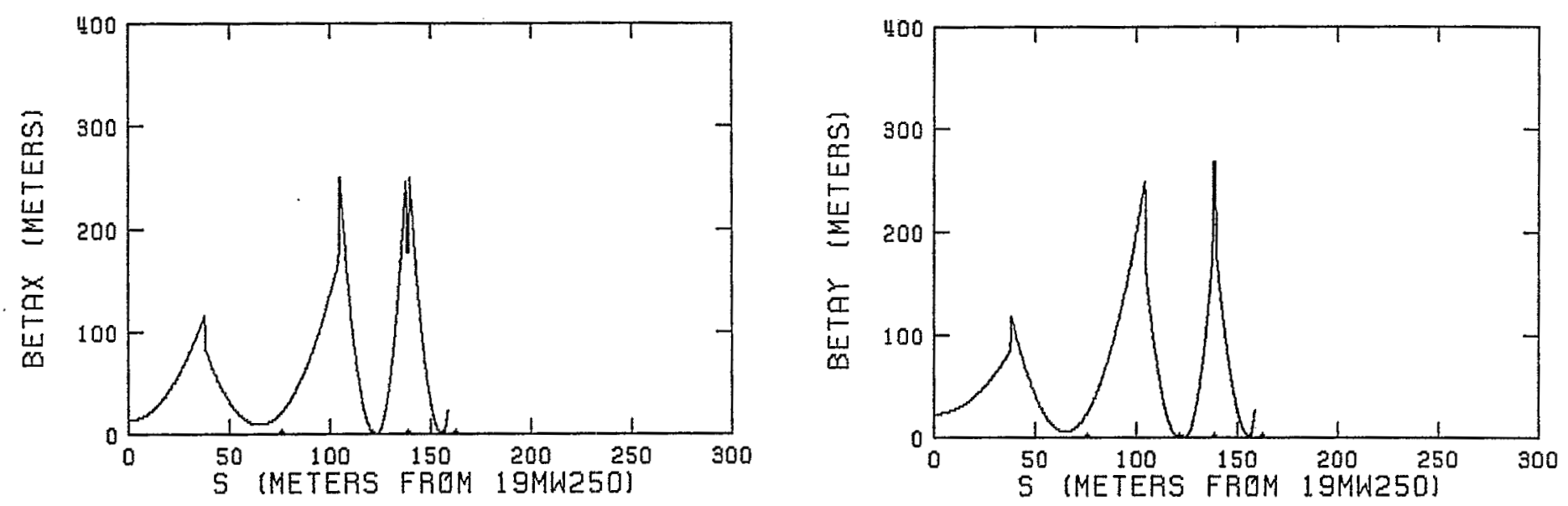

Figures 34 and 35: Horz and Vert $\beta(s), I=5.0$.
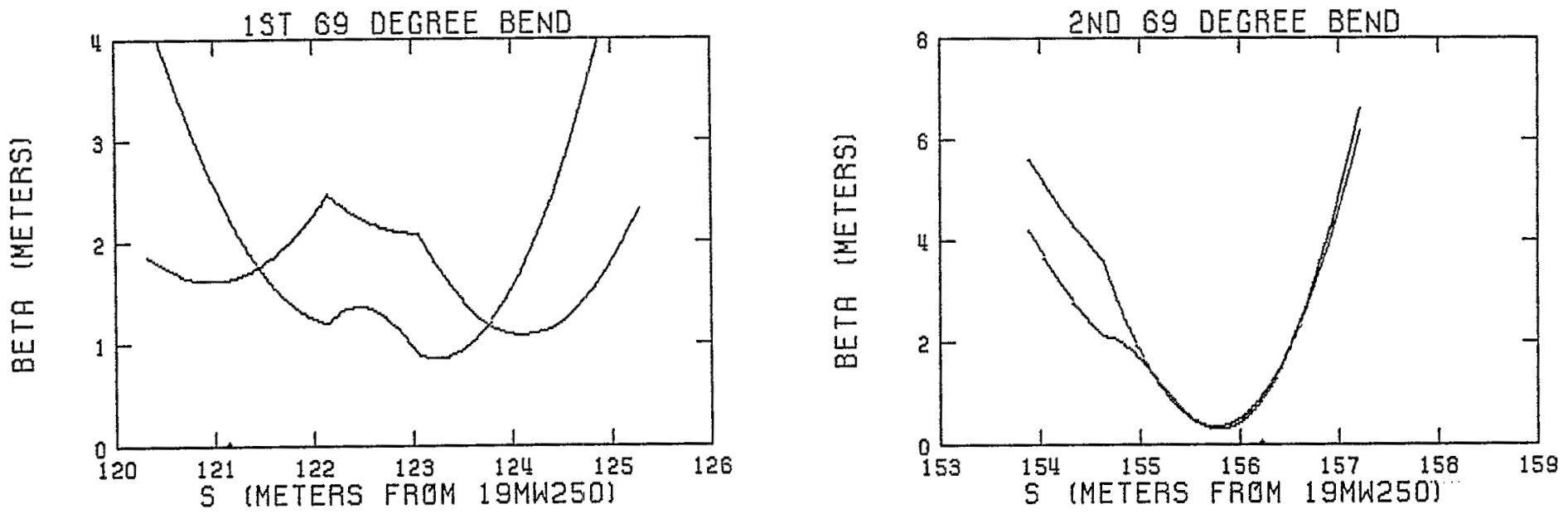

Figures 36 and 37: $\beta(s)$ near 1st and 2 nd $69^{\circ}$ bends, $I=5.0$. 
FIGURES 38-41 (HITL Sections 20-22)
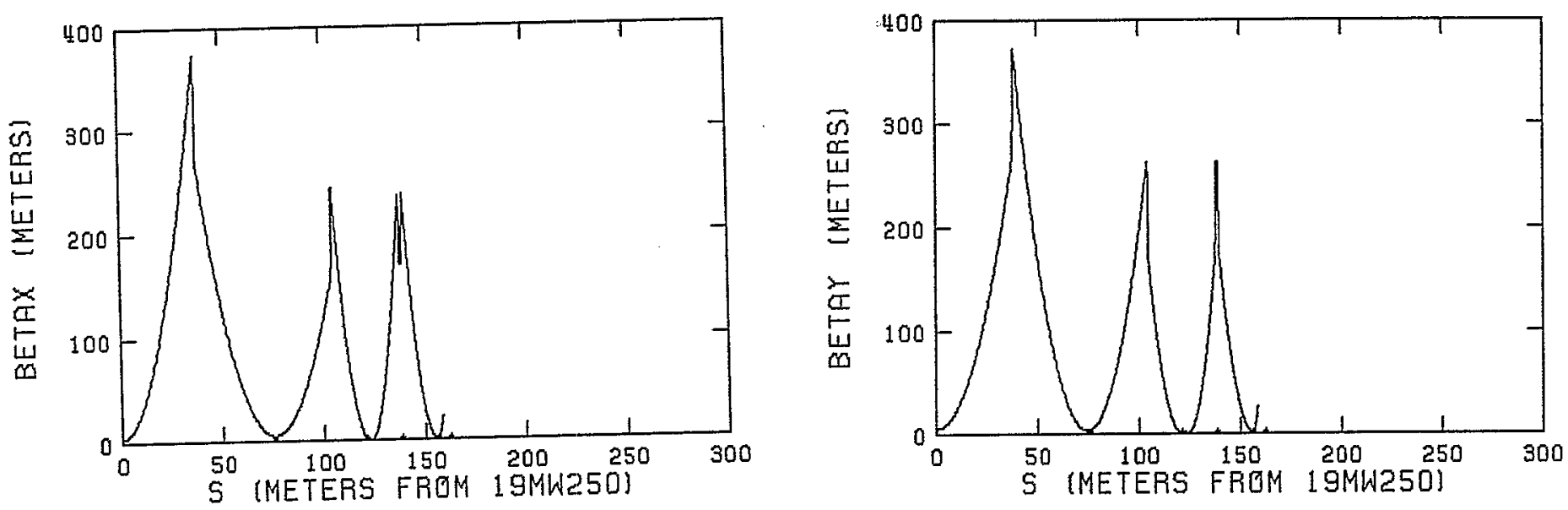

Figures 38 and 39: Horz and Vert $\beta(s), I=5.52$.
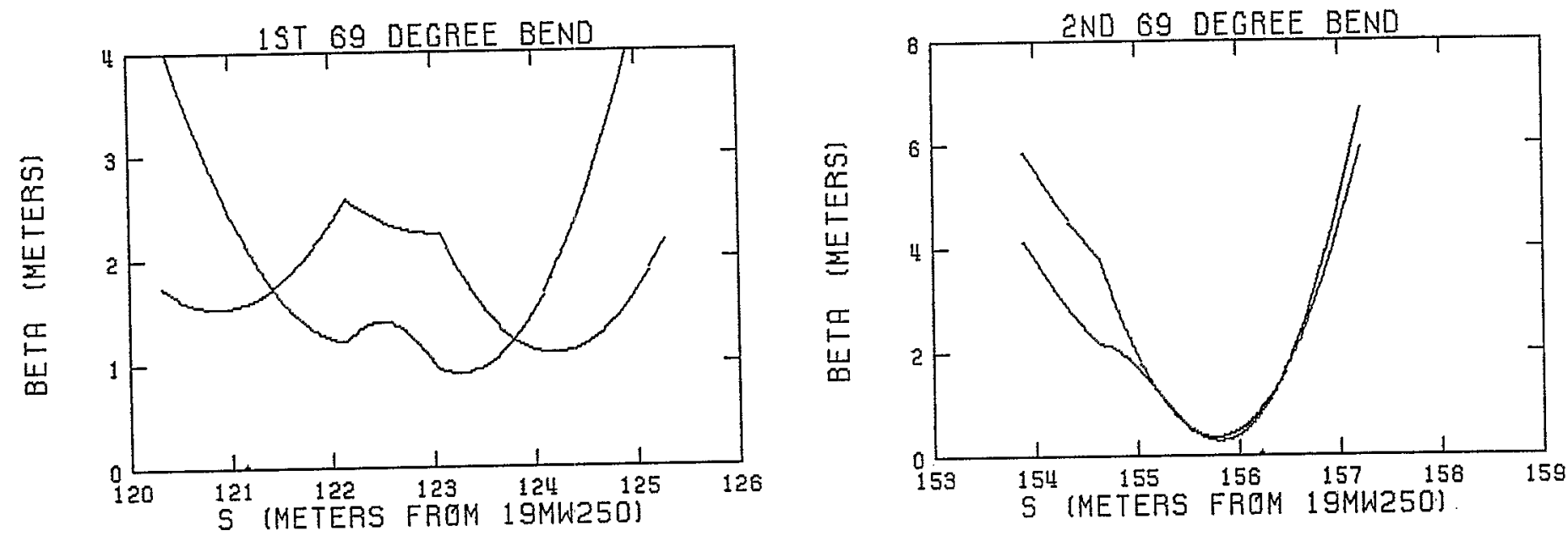

Figures 40 and $41: \beta(s)$ near 1st and 2 nd $69^{\circ}$ bends, $I=\mathbf{5 . 5 2}$. 
FIGURE 42

HITL SECTION 23

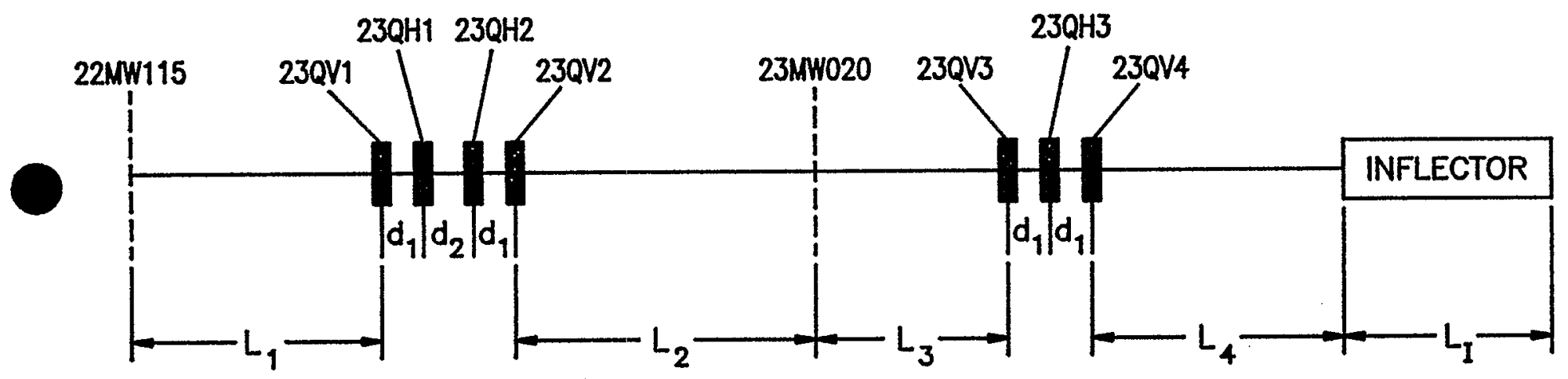

Figure 42. HITL section 23. 

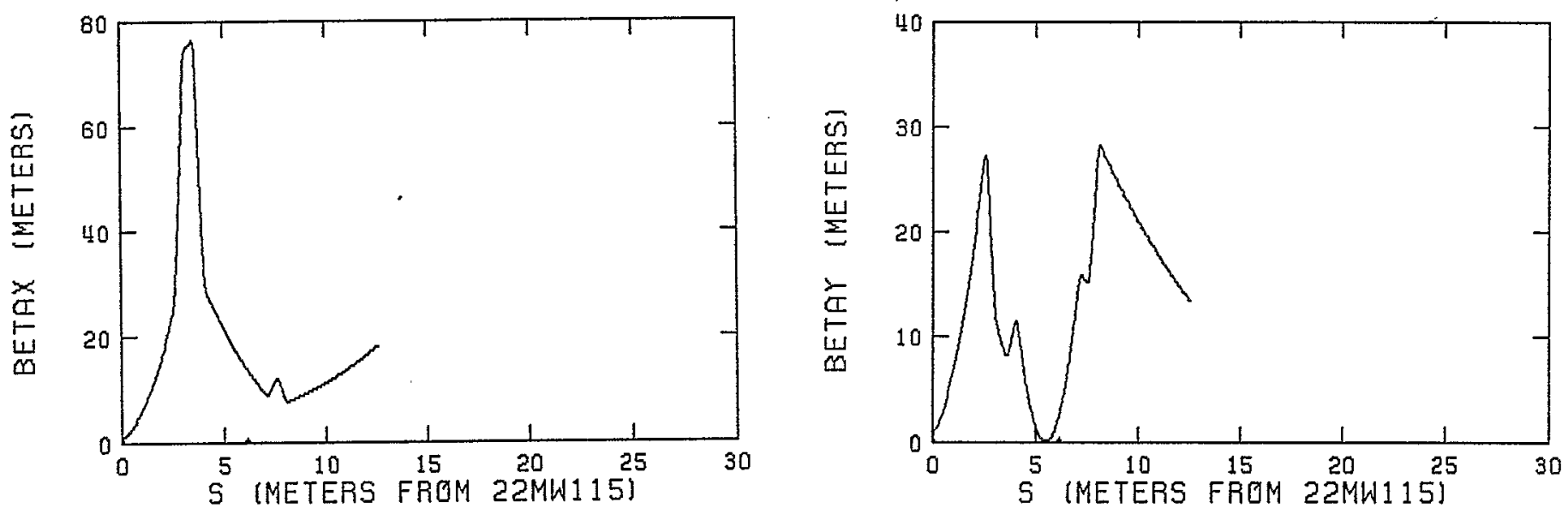

Figures 43 and 44: Sect. 23 Horz and Vert $\beta(s), I=5.0$.
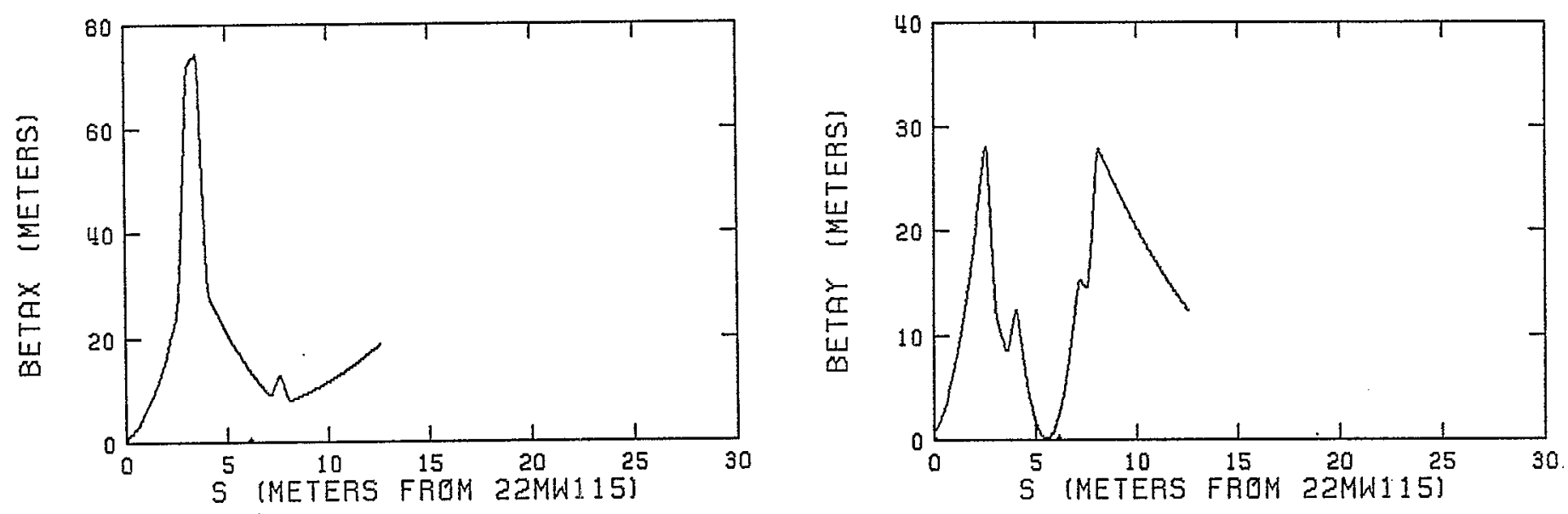

Figures 45 and 46: Sect. 23 Horz and Vert $\beta(s), I=5.52$. 